\title{
Estimación de los factores condicionantes de la adquisición de competencias académicas en América Latina en presencia de endogeneidad
}

\author{
Geovanny Castro Aristizabal, Gregorio Giménez y \\ Domingo Pérez Ximénez-de-Embún ${ }^{1}$
}

\section{Resumen}

En este artículo se identifican los principales condicionantes de la adquisición de competencias en América Latina. Se establecen como características individuales y familiares la condición de no repetidor, el sexo, la cantidad de libros en el hogar y la educación de la madre. En el caso de los factores escolares, los resultados son más heterogéneos entre los países. Los principales factores serían la asistencia a colegio privado, la cantidad de alumnos por aula, la calidad de los materiales educativos, y el mayor tamaño y autonomía de los centros. Las características de las escuelas explican la mayor parte de la variabilidad de los resultados, seguidas por las características familiares y las individuales. Las características de las escuelas desempeñan un papel especialmente relevante en la Argentina, el Brasil y Costa Rica; las familiares, en Chile, Colombia y el Perú; y las individuales, en Colombia y México.

\section{Palabras clave}

Desarrollo de capacidad, rendimiento escolar, educación, calidad de la educación, evaluación, indicadores educativos, investigación educativa, América Latina

\section{Clasificación JEL}

C29, 121, 124, 128, 129

\section{Autores}

Geovanny Castro Aristizabal es Profesor e Investigador del Departamento de Economía de la Pontificia Universidad Javeriana de Cali (Colombia). Correo electrónico: gcastro@javerianacali.edu.co.

Gregorio Giménez es Profesor e Investigador en el Departamento de Economía Aplicada de la Universidad de Zaragoza (España). Correo electrónico: gregim@ unizar.es.

Domingo Pérez Ximénez-de-Embún es Profesor e Investigador en el Departamento de Análisis Económico de la Universidad de Zaragoza (España). Correo electrónico: dpxe@unizar.es.

Los autores agradecen la ayuda financiera recibida del Banco Santander, la Universidad de Zaragoza y la Pontificia Universidad Javeriana de Cali (Colombia). También agradecen los comentarios realizados por los evaluadores anónimos y por Macarena Iranzo, que han contribuido a mejorar la versión final del artículo. Todos los posibles errores son responsabilidad única de los autores. 


\section{Introducción}

En los países de América Latina participantes en el Programa Internacional para la Evaluación de los Estudiantes (PISA) 2012 se ha asumido el reto de mejorar la calidad educativa. Así, se ha aumentado el gasto público por alumno y se han realizado cambios legislativos para garantizar a la población más desfavorecida el derecho a la educación. Aunque no todos han avanzado al mismo ritmo, en general sí han experimentado mejoras al ampliar la oferta educativa construyendo escuelas y creando nuevas plazas docentes.

Ello ha dado lugar a un aumento de los años promedio de escolaridad y a una mejora de los resultados en las distintas pruebas internacionales en las que han participado (CIPPEC, 2011). Al comparar los resultados del Segundo Estudio Regional Comparativo y Explicativo (SERCE 2006) con los del Tercer Estudio Regional Comparativo y Explicativo (TERCE 2013), en general se observa un incremento significativo de la puntuación obtenida por los estudiantes de tercer y sexto grado en las tres áreas evaluadas, sobre todo en matemáticas (Rivas, 2015)². Sin embargo, en educación secundaria, el rendimiento de la región latinoamericana sigue siendo relativamente bajo si adoptamos una perspectiva global. Es decir, en relación con las tres competencias evaluadas, los ocho países de América Latina que participaron en el PISA 2012 obtuvieron resultados que los situaron en los 20 últimos puestos entre las 65 economías participantes ${ }^{3}$.

En este sentido, pese a que en términos generales hay un gran número de trabajos empíricos sobre economía de la educación en los que se ha tratado de arrojar luz sobre los factores que condicionan el rendimiento escolar o la adquisición de competencias, en lo que respecta a América Latina, estos trabajos son relativamente escasos.

Cabe destacar que en estas investigaciones se emplea la función de producción educativa (FPE) y se la estima a través de diferentes metodologías. En particular, ha ganado espacio el uso de técnicas multinivel (o de modelos jerárquicos), ya que estas han sido ampliamente aceptadas como unas de las más idóneas para estudiar y analizar los datos educativos. Esto se debe a que las características del estudiante y del centro escolar (insumos (inputs) de la FPE) están anidadas a través de su desempeño escolar, es decir, presentan una estructura jerárquica (Gaviria y Castro, 2005).

Sin embargo, en estos modelos jerárquicos se supone que no hay correlación entre las variables independientes y el error del modelo. No obstante, no todos los insumos de la FPE son estadísticamente exógenos (correlación con el error igual a cero), ya que en el modelo se omiten variables que no se pueden medir de forma directa. Por esta razón, pueden estar correlacionadas con el error y causar un problema de endogeneidad (Hanushek y Woessmann, 2011). Dada la existencia de este problema, la aplicación de las técnicas multinivel arroja coeficientes inconsistentes y sesgados, motivo por el cual en la literatura se sugiere emplear el método de las variables instrumentales (Greene, 2012). A pesar de ello, este método ha sido escasamente empleado en economía de la educación (véase Hanushek y Woessmann, 2011) y no ha sido utilizado en el análisis del rendimiento escolar en América Latina.

Teniendo en cuenta lo expuesto, este artículo tiene por objetivo identificar los factores condicionantes en la adquisición de competencias en América Latina. Para ello, se emplea la información de los países participantes en el PISA 2012 en las tres áreas evaluadas. Además, se estima la FPE mediante el método generalizado de momentos ya que, por un lado, se ha detectado el problema de la endogeneidad y, por el otro, no es posible la agregación a nivel de país debido a la falta de pesos relativos regionales en la muestra seleccionada.

2 Corresponden a las evaluaciones que el Laboratorio Latinoamericano de Evaluación de la Calidad Educativa (LLECE) de la UNESCO aplica a países de América Latina con el propósito de medir los logros en aprendizaje de los estudiantes de la región en las competencias de lectura, matemáticas y ciencias.

3 Los países de América Latina participantes son: Argentina, Brasil, Chile, Colombia, Costa Rica, México, Perú y Uruguay. 
El artículo se encuentra estructurado de la siguiente manera. Después de esta introducción, aparece un segundo apartado en el que se hace un repaso de la literatura relativa a los factores determinantes de la adquisición de competencias en países en vías de desarrollo. En el tercero se recoge la parte metodológica, en la que se expone el modelo empleado y se describen las variables incluidas en él y las técnicas econométricas usadas para su análisis. El cuarto contiene los resultados del análisis empírico, que a través de la estimación de la FPE revela que los principales factores determinantes del éxito escolar son la condición de no repetidor, el sexo, la cantidad de libros en el hogar y el nivel educativo de la madre. Por último, en el quinto apartado se recogen las conclusiones.

\section{La literatura sobre el rendimiento académico en América Latina}

En los primeros trabajos realizados sobre calidad educativa y rendimiento escolar (Alexander y Simmons, 1975; Jencks, 1972, y Coleman y otros, 1966) se concluyó que los antecedentes familiares determinan en gran medida el desempeño académico de los estudiantes. Más recientemente, en la misma línea, Woessmann (2010) sostuvo que el rendimiento escolar está fuertemente relacionado con los antecedentes familiares y débilmente con las características de la escuela. En el ámbito de América Latina, Cervini (2012) analizó el efecto escuela sobre el desempeño académico. Con ese fin, aplicó modelos multinivel bivariados (en tres niveles: país, alumno y escuela), empleó los resultados del Segundo Estudio Regional Comparativo y Explicativo (SERCE 2006) en matemáticas y lectura, controló por factores extraescolares y encontró que el efecto escuela sobre el rendimiento en estas pruebas fue muy bajo.

En las últimas décadas, los estudios sobre educación y desempeño académico han aumentado considerablemente, aunque los resultados obtenidos son heterogéneos. De una parte, hay investigaciones en las que se concluye que las características del estudiante o las de su entorno familiar son las que se constituyen como el factor más relevante. Sin embargo, existe evidencia empírica que señala que los factores asociados a los centros escolares y a las instituciones son los aspectos que más influyen sobre el desempeño escolar.

Los resultados encontrados respecto de países de América Latina también reflejan esta ambigüedad. Por un lado, algunos muestran una fuerte asociación entre el desempeño académico y las características individuales y familiares de los estudiantes, y, por otro, en diversos estudios se concluye que son los aspectos escolares los factores más importantes. No obstante, cabe destacar que el número de investigaciones en las que se llega a esta segunda conclusión es relativamente menor (véase el cuadro 1).

En el caso de las características individuales, el sexo, la repetición de curso y la raza se erigen como los factores que más influyen en el desempeño escolar. De esta manera, se observa lo siguiente: i) en promedio, las mujeres obtienen mejores resultados en lectura y los hombres lo hacen en matemáticas y ciencias; ii) la condición de repetidor de curso afecta negativamente la puntuación media obtenida en cada una de las áreas evaluadas, y iii) los estudiantes de raza negra tienen mayores desventajas que los estudiantes de otras razas y, por tanto, obtienen peores resultados.

Respecto a los factores familiares, los trabajos empíricos existentes sobre la situación en los países de América Latina en cuanto al rendimiento escolar señalan que el conjunto de características familiares y el entorno condicionan de forma favorable el desempeño de los estudiantes Lo mismo ocurre con la educación de los padres, en particular, el nivel educativo de la madre. 
Cuadro 1

América Latina: factores determinantes del rendimiento escolar

\begin{tabular}{|c|c|c|c|c|}
\hline Factor & & Autor(es) & País o región & Datos \\
\hline \multirow{3}{*}{ 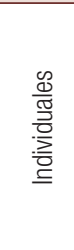 } & $\operatorname{Sexo}(+,-)$ & $\begin{array}{l}\text { Cárcamo y Mola (2012); } \\
\text { Woessmann (2010); Vegas } \\
\text { y Petrow (2007) }\end{array}$ & $\begin{array}{l}\text { Colombia; Argentina; } \\
\text { países latinos en PISA }\end{array}$ & SABER11 2009; PISA 2000-2003 \\
\hline & Rezago (-) & $\begin{array}{l}\text { Oreiro y Valenzuela (2013); Méndez } \\
\text { y Zerpa (2011); Post (2011) }\end{array}$ & $\begin{array}{l}\text { Uruguay; Uruguay y Chile; Chile, } \\
\text { Colombia, Ecuador y Perú }\end{array}$ & $\begin{array}{l}\text { PISA 2003; PISA 2006; } \\
\text { SERCE 2006 y Encuestas } \\
\text { Regionales de Empleo }\end{array}$ \\
\hline & Raza (-) & $\begin{array}{l}\text { Marteleto (2012); Viáfara } \\
\text { y Urrea (2006) }\end{array}$ & Brasil, Colombia & $\begin{array}{l}\text { Encuesta nacional } 1982 \\
\text { y 2007; ENH } 2000\end{array}$ \\
\hline \multirow{2}{*}{ 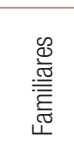 } & Conjunto $^{\mathrm{a}}(+)$ & $\begin{array}{l}\text { Thieme, Prior y Tortosa-Ausina } \\
\text { (2013); Donoso y Hawes (2002); } \\
\text { Vivas, Correa y Domínguez (2011) }\end{array}$ & Chile; Chile; Colombia & $\begin{array}{l}\text { SIMCE 2008; SIMCE } \\
\text { 2000; ECV2003 }\end{array}$ \\
\hline & $\begin{array}{l}\text { Educación } \\
\text { padres (+) }\end{array}$ & $\begin{array}{l}\text { Ayala, Marrugo y Saray } \\
\text { (2011); Sánchez (2011) }\end{array}$ & Colombia & SABER11 2010 \\
\hline \multirow{2}{*}{ 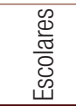 } & Profesores (+) & $\begin{array}{l}\text { Banco Mundial (2005); León, } \\
\text { Manzi y Paredes (2004) }\end{array}$ & México; Chile & $\begin{array}{l}\text { PISA 2003; Sistema } \\
\text { Evaluación Docente }\end{array}$ \\
\hline & Jornada $(+)$ & Bonilla (2011) & Colombia & SABER11 2009 \\
\hline
\end{tabular}

Fuente: Elaboración propia.

Nota: SABER11 hace referencia a los resultados obtenidos en las pruebas aplicadas a los estudiantes del último curso de bachillerato en el Sistema Nacional de Evaluación de la Calidad de la Educación. PISA se refiere al Programa Internacional para la Evaluación de los Estudiantes. SERCE significa Segundo Estudio Regional Comparativo y Explicativo. ENH es la Encuesta Nacional de Hogares. SIMCE es el Sistema de Medición de la Calidad de la Educación. ECV es la Encuesta de Calidad de Vida. El símbolo entre paréntesis que figura junto a cada uno de los factores se refiere a la relación entre el desempeño y el factor. Cuando el género se mide con un variable ficticia (dummy) con valor 1 si es mujer, 0 si es hombre, el efecto sobre la puntuación en lectura es (+) y en matemáticas (-).

a Hace referencia a las características socioeconómicas.

Por último, en cuanto a las características escolares, la calidad de los profesores y el tipo de jornada escolar (mañana o tarde) parecen ser determinantes para el desempeño académico de los estudiantes (véase el cuadro 1). Cabe destacar que los trabajos en los que se estiman funciones de producción educativa en América Latina son todavía pocos y, en todo caso, no suelen incorporar técnicas econométricas avanzadas. En este trabajo se tienen en cuenta dichas limitaciones y se trata de incorporar una nueva perspectiva. Para ello, se utilizan técnicas de imputación para datos sin registro y se hace uso de estimadores robustos e insesgados ante la presencia de endogeneidad con origen en la doble causalidad entre variables endógenas y exógenas. El uso de estas técnicas permite observar, respecto a la muestra con la que trabajamos, el mayor peso que tendrían las características familiares en la explicación de la variabilidad de los rendimientos. También apunta a la existencia de brechas de rendimiento entre las instituciones públicas y privadas.

\section{Metodología}

\section{Modelo y descripción de los insumos de la función de producción educativa}

La función de producción educativa (FPE) posee las mismas características que una función de producción típica. En ella se relaciona el producto (output), es decir, los resultados académicos o las puntuaciones de los alumnos, con los insumos, a saber, un conjunto de variables asociadas al estudiante y al centro escolar. Nosotros seguimos a Hanushek, Link y Woessmann (2013), y a Hanushek y Woessmann (2012 y 2011), y trabajamos con la siguiente FPE:

$$
P M_{i j}^{p}=\beta_{0}+\sum_{i=1}^{m} \beta_{i} C E_{i}^{p}+\sum_{i=m+1}^{h} \beta_{i} C F_{i}^{p}+\sum_{i=h+1}^{w} \beta_{i} F E_{i}^{p}+\varepsilon_{i}
$$


En ella, $P M_{i j}^{p}$ corresponde al valor medio de los cinco valores plausibles (resultados) del estudiante $i$ en la competencia $j$ del país $p$ (véase OCDE, 2014). $\varepsilon_{i}$ hace referencia al término aleatorio de error del modelo, en el que se incorporan las variables que no son directamente observables o medibles, como la capacidad de aprender, las habilidades innatas del estudiante o el "efecto

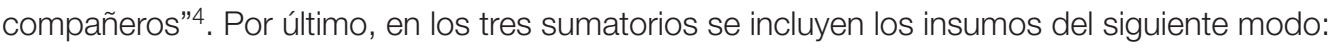

- $\quad$ En el primer sumatorio se encuentran las características del estudiante (CE). Se han creado las siguientes variables:

i) La variable sexo, que toma el valor de 1, si el estudiante es mujer, o de 0, si es hombre. Mide las desigualdades entre ambos sexos por competencias (véanse Woessmann, 2010, en relación con lectura; García, Hidalgo y Robles, 2010, y Mullis y otros, 2007, en cuanto a matemáticas, y Vegas y Petrow, 2007, en lo que respecta a ciencias).

ii) La variable norepetidor, a la que se le asigna el valor de 1 , si el estudiante ha repetido al menos un curso, y de 0 , en caso contrario. Se emplea para medir el impacto de lo que en la literatura se define como "rezago escolar" (Méndez y Zerpa, 2011; Hong y Yu, 2007).

iii) La variable esfuerzo, que toma el valor de 1, si el alumno busca información adicional para aclarar un tema que estudia y no comprende, y de 0 , si no realiza dicha búsqueda.

iv) La variable disciplina, a la que se le da el valor de 1 , si los estudiantes están atentos a lo que dice el profesor en la mayoría de las clases, y de 0, si no es así (Post, 2011; Cervini, 2003).

- $\quad$ El segundo sumatorio reúne las características familiares (CF), que permiten medir el impacto del estatus socioeconómico y cultural del hogar sobre la adquisición de competencias. Para ello, se generan, específicamente, las siguientes variables:

i) libros, cuyo valor es de 1, si la cantidad de libros en el hogar es mayor de 200, y de 0, si es menor (Woessmann y otros, 2007).

ii) educamadre y educapadre, que hacen referencia al nivel mínimo de estudios de la madre y el padre. Seguimos a Hanushek y Luque (2003) y, si es bachiller, asignamos un valor de 1 y, si no lo es, de 0.

iii) emppadre, que toma el valor de 1, si el tipo de contrato del padre es de medio tiempo o tiempo completo, y de 0, en caso contrario (Hanushek y Woessmann, 2011; Woessmann y otros, 2007).

- El último sumatorio recoge los factores escolares (FE), que son los siguientes:

i) La titularidad del centro escolar, que permite detectar las brechas educativas entre los colegios públicos y los privados. Así, se crea la variable publico, a la que se le da el valor de 1 , si el colegio es público, y de 0, si es privado (Gamboa y Waltenberg, 2012).

ii) La proporción de alumnos por profesor (STRATIO), la cantidad de estudiantes matriculados en el centro escolar (SCHSIZE) y la calidad de los materiales educativos (SCMATEDU), que se incorporan como indicadores indirectos (proxy) del gasto educativo del centro escolar (Hanushek, 2011; Vignoles y otros, 2000).

\footnotetext{
4 El "efecto compañeros" (peer effects) se encuentra en esta parte residual de la función porque plantea ciertas cuestiones de naturaleza teórica y empírica que dificultan su medición. Murnane (1981) pone de manifiesto la dificultad de identificar el grupo relevante de estudiantes con los que se relaciona el alumno (¿los de su misma clase, los del mismo curso o los del mismo centro escolar?). Este autor también destaca la dificultad que existe para determinar los rasgos de este grupo de referencia que pueden realmente incidir en el rendimiento escolar de cada alumno (¿el nivel socioeconómico del alumnado, su composición racial, su nivel académico, el sexo?). Por otra parte, otros autores, como Brunello y Rocco (2008) o Angrist (2014), argumentan que existen problemas de naturaleza empírica que limitan estos resultados. Se trata, principalmente, de la existencia de sesgos de selección, correlaciones espurias y problemas de endogeneidad.
} 
iii) La variable ficticia (dummy) autonomia, que se construye a partir de lo propuesto por Hindrinks y otros (2010), y toma el valor de 1, si el director y los profesores son autónomos en la toma de decisiones relacionadas con las instancias importantes de la institución, y de 0 , en caso contrario.

\section{Fuentes de información y tratamiento de los datos sin registro}

La información con la que se construyen los insumos de la FPE y con la que se realizan las estimaciones del modelo (1) se toma de PISA 2012, disponible en OCDE (2015). Se tiene en cuenta la información tanto de estudiantes como de escuelas de los países latinoamericanos que participaron en las pruebas PISA 2012. En total, se obtienen 90.799 observaciones de estudiantes divididos en 3.722 escuelas. Estas observaciones se encuentran distribuidas de la siguiente forma: 9.073 estudiantes y 352 escuelas de Colombia, 5.908 y 226 de la Argentina, 19.204 y 839 del Brasil, 6.856 y 221 de Chile, 4.602 y 193 de Costa Rica, 33.806 y 1.471 de México, 6.035 y 240 del Perú, y 5.315 y 180 del Uruguay. Estas observaciones son estadísticamente representativas de la población de cada uno de los países estudiados (OCDE, 2015).

Sin embargo, esta base de datos contiene numerosos valores omitidos, esto es, datos que corresponden a la información sin registro debido a la falta de respuesta a los cuestionarios que deben realizar los estudiantes y directores de los centros escolares. Esto puede dar lugar a sesgos en la inferencia estadística, razón por la que es necesario reemplazar estos datos ausentes utilizando métodos de imputación (Medina y Galván, 2007). Así, en este trabajo se sigue la propuesta de los autores citados y se aplica el método hot-deck a aquellas variables que superan el 10\% de valores omitidos en la base de datos. Este método no paramétrico, de acuerdo con Durrant (2009), mantiene la distribución de probabilidad de las variables imputadas. Por lo tanto, es más eficiente que los métodos de imputación múltiple.

En el cuadro 2 se muestra la media y la desviación estándar de las variables empleadas en la FPE. Cabe destacar que los promedios obtenidos por los estudiantes de los países latinoamericanos objeto de nuestro estudio en lectura, matemáticas y ciencias en el PISA 2012, que se sitúan en torno a los 500 puntos en cada una de las áreas, resultan insatisfactorios cuando se los compara con los de la OCDE. Esto se refleja en las bajas posiciones que ocupan dichos países en la clasificación de las 65 economías participantes (34 de la OCDE y 31 asociadas), que se observan a la derecha del promedio en el cuadro.

La heterogeneidad que se observa en los resultados se puede deber a los insumos que se contemplan en la FPE y se recogen en el cuadro, pero también a factores adicionales. Así, hay que tener en cuenta que, si el rendimiento escolar está condicionado por las circunstancias socioeconómicas, las de los países latinoamericanos distan de las del promedio de la OCDE. En el gráfico 1 se muestra la relación entre la renta y el desempeño en Matemáticas (línea discontinua negra), respecto a las 65 economías participantes en el PISA. Esta relación sugiere que las diferencias en la renta pueden explicar un $21 \%$ de la variación entre los resultados escolares de las economías que participaron. Por tanto, los países más ricos tienen una clara ventaja.

Además, esta relación es más fuerte si se tienen en cuenta exclusivamente los ocho países latinoamericanos objeto de nuestra investigación (línea discontinua roja), ya que un 59\% de la variabilidad entre los resultados de los países puede justificarse por sus diferencias en materia de renta. La renta promedio en la región (13.175 dólares) se encuentra entre los extremos de Chile (17.312 dólares ) y el Perú (9.350 dólares). 


\section{Cuadro 2}

América Latina (8 países): medias y desviaciones estándar de las variables incluidas en la función de producción educativa, 2012a

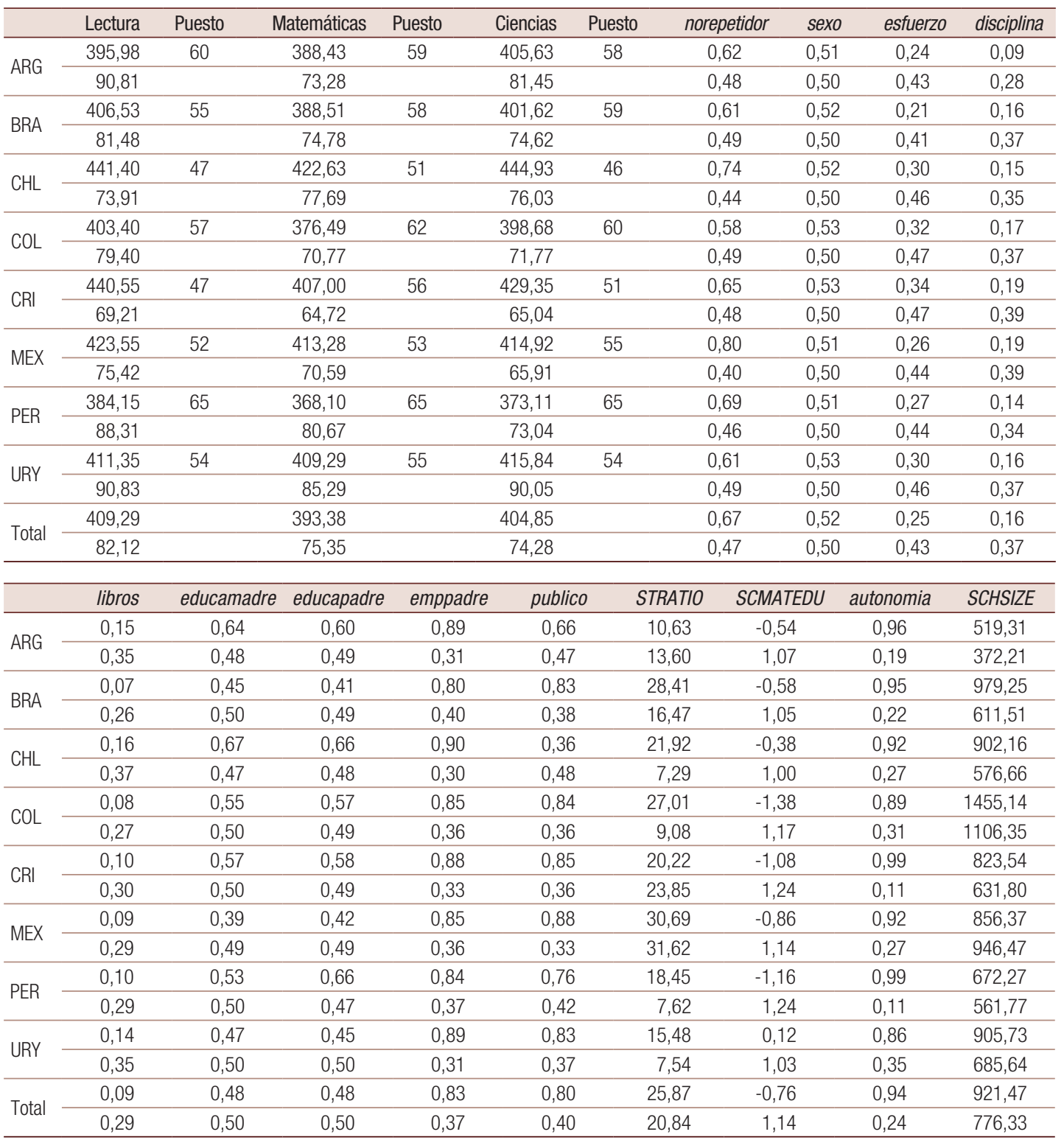

Fuente: Elaboración propia, sobre la base de datos de la Organización de Cooperación y Desarrollo Económicos (OCDE) sobre la prueba PISA 2012.

Nota: El total se ha elaborado con los datos promedio de todos los estudiantes de América Latina. El puesto se refiere al lugar que ocupa entre los países participantes en la prueba PISA.

a La media se indica en la línea superior y la desviación estándar en la línea inferior. 


\section{Gráfico 1}

Resultados en matemáticas y PIB per cápita de las 65 economías que participaron en las pruebas PISA, 2012

(En notas medias y dólares corrientes corregidos por paridad del poder adquisitivo (PPA))

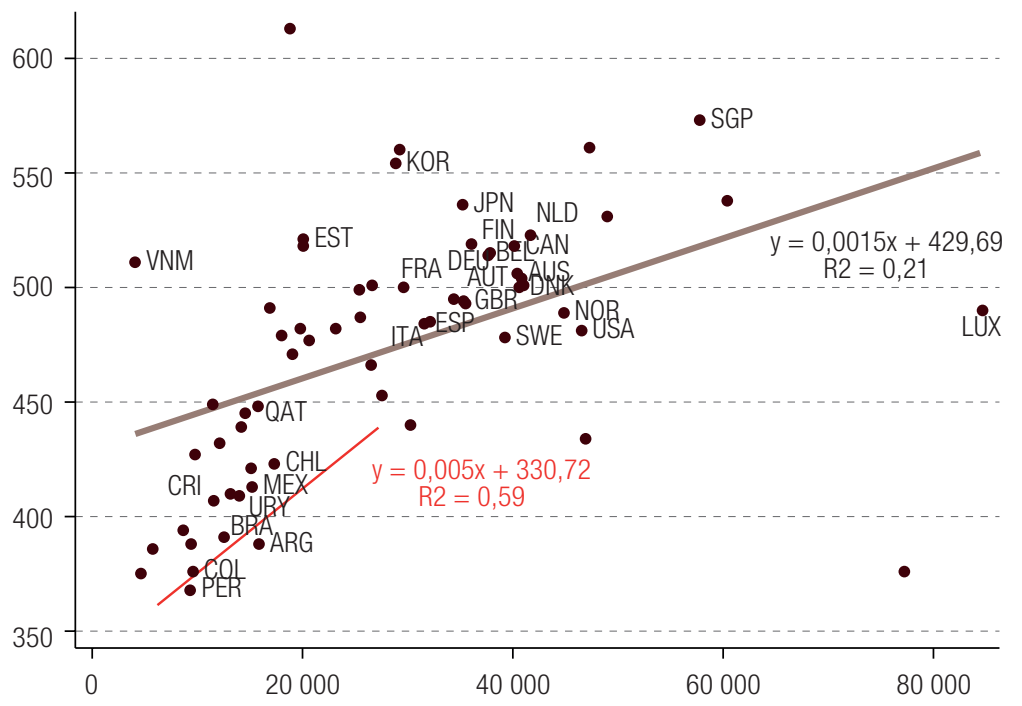

Fuente: Elaboración propia, sobre la base de datos de la Organización de Cooperación y Desarrollo Económicos (OCDE) sobre la prueba PISA 2012

Al igual que las diferencias en la renta, la distribución de esta también constituye un factor limitativo. Es decir, en América Latina, la renta se distribuye de un modo especialmente inequitativo y la desigualdad afecta gravemente a los niños y adolescentes. Los países que tienen una mayor proporción de estudiantes con problemas socioeconómicos tienen que afrontar mayores retos formativos. Esta situación se refleja en el gráfico 2, donde se muestra la relación entre los resultados en matemáticas y el porcentaje de alumnos que se encuentran en circunstancias socioeconómicas adversas. Estas tendrían un efecto negativo en el desempeño escolar (línea discontinua negra), ya que el índice se asocia a un $24 \%$ de la variabilidad en los resultados en matemáticas. La relación es más intensa en el contexto de los países latinoamericanos (línea discontinua roja). Un 32\% de la variabilidad en los resultados de dichos países se asocia a las diferencias relacionadas con las circunstancias adversas. Un 51\% de los estudiantes latinoamericanos vivían en entornos desfavorables. En el Perú se presentaba la mayor proporción de estudiantes en dicha situación $(59,9 \%)$ y en Chile la menor (37,9\%).

Sin embargo, aunque el nivel de renta y el porcentaje de alumnos que se encuentran en circunstancias socioeconómicas adversas son factores limitativos importantes, estos no justifican las diferencias entre los resultados de los países latinoamericanos y los del resto de las economías que participaron en la prueba PISA. En efecto, en los gráficos 1 y 2, los países latinoamericanos se encuentran por debajo de la recta de regresión minimocuadrática, por lo que puede interpretarse que su resultado promedio en la prueba de matemáticas está por debajo de lo esperado, teniendo en cuenta su nivel de renta y el porcentaje de alumnos en entornos desfavorables. Es decir, de acuerdo con la línea de regresión del gráfico 1, el resultado esperado en relación con los países latinoamericanos sería de 449 puntos, esto es, 58 puntos por encima de los realmente obtenidos. Del mismo modo, de acuerdo con la recta de regresión del gráfico 2, el resultado esperado sería de 440 puntos: 47 puntos por encima de lo obtenido. 


\section{Gráfico 2}

Resultados en matemáticas y proporción de estudiantes en circunstancias socioeconómicas adversas en las 65 economías participantes en la prueba PISA, 2012

(En notas medias y en porcentajes)

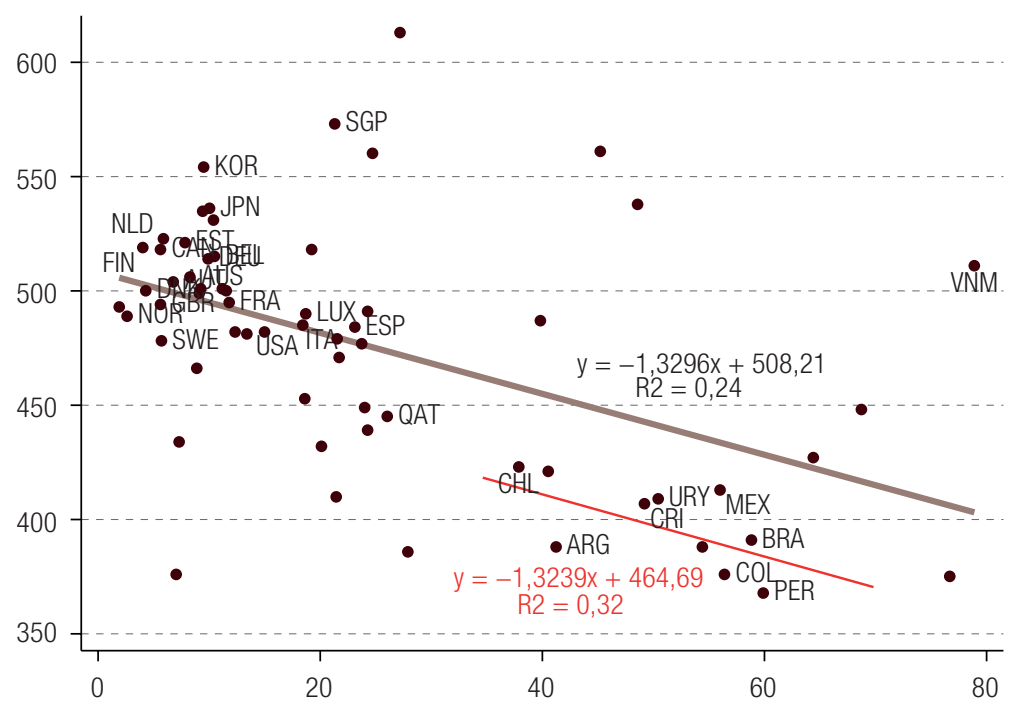

Fuente: Elaboración propia, sobre la base de datos de la Organización de Cooperación y Desarrollo Económicos (OCDE) sobre la prueba PISA 2012.

\section{El problema de la endogeneidad en la función de producción educativa}

Los insumos de la FPE son susceptibles de estar correlacionados con el término de perturbación de error del modelo (1). Por tanto, la correlación entre ellos puede ser diferente de cero debido a la omisión de variables. Puesto que algunos factores que determinan el rendimiento no son directamente medibles, no todos los insumos son exógenos desde el punto de vista estadístico (Hanushek y Woessmann, 2011). Por ejemplo, la condición de no repetidor (insumo correspondiente a las características individuales) puede estar asociada con la capacidad de aprender, las habilidades innatas del estudiante o la motivación. Estos factores no se pueden observar de forma directa, por ello se los incluye en el error, lo que conduce a una correlación distinta de cero entre el hecho de ser repetidor y la perturbación ${ }^{5}$.

Al existir dicha correlación en al menos uno de los insumos de la FPE, se presenta el problema de la endogeneidad. Por tanto, las estimaciones a través de los mínimos cuadrados ordinarios (MCO) o de los modelos jerárquicos (multinivel) no son convenientes. Con todo, los modelos multinivel se siguen usando ampliamente en educación, porque ofrecen la ventaja de que permiten sortear los posibles sesgos de selección en los centros educativos. Mientras los modelos clásicos asumen efectos fijos, esto es, efectos comunes a todos los individuos, los modelos multinivel están compuestos por dos partes diferenciadas: una común y fija para todos los contextos, y una segunda que varía y se estima en función de cada nivel. De esta manera, al modelizar de forma simultánea múltiples unidades de análisis, es posible estimar con precisión el aporte de las variables de cada uno de los niveles (escuelas en PISA) al rendimiento académico del alumno.

\footnotetext{
5 Asimismo, si se consideran los aspectos escolares, el tamaño de la clase y del colegio, la titularidad del centro y la autonomía pueden depender, a su vez, de la política educativa y las decisiones que se toman en las instituciones administrativas, como los ministerios de educación, o de las disposiciones mismas de los directores y profesores del centro escolar. Como consecuencia, se puede tener una correlación distinta de cero entre estos factores (inobservables e incluidos en el error) y los insumos escolares.
} 
Sin embargo, como hemos apuntado, los coeficientes estimados en los modelos multinivel en presencia de endogeneidad serán sesgados e inconsistentes (Wooldridge, 2010). Si existe endogeneidad, la literatura sugiere aplicar las metodologías del emparejamiento por puntaje de propensión (propensity score matching (PSM)) o la de las variables instrumentales (VI). Estos métodos serían consistentes y también permitirían lidiar con el problema de los sesgos de selección. La principal diferencia entre los métodos del PSM y las VI es que el PSM se utiliza habitualmente para comparar grupos: uno de ellos recibe tratamiento y el otro no. Además, en el PSM se emplean factores observables para construir los pesos en las estimaciones, mientras que el método de las VI se basa en el empleo de instrumentos a partir de factores no medidos o no observados. Así, la ventaja de utilizar las $\mathrm{VI}$ es que se tiene en cuenta la existencia de estos factores no observados, correlacionados con los resultados escolares. Esto es de vital importancia cuando se trabaja con la FPE, dado que es inevitable que en los insumos utilizados no se recojan todos los elementos que influyen en los resultados.

El problema del método de las VI es que no ofrece estimadores eficientes si realmente no existe endogeneidad, por lo que se debe comprobar la presencia de esta. Además, puede ser difícil encontrar instrumentos válidos que satisfagan las condiciones necesarias, esto es, que se correlacionen con los insumos de la FPE pero no directamente con los resultados escolares. A fin de detectar el problema de la endogeneidad, en este trabajo se utiliza el estadístico de prueba del método generalizado de momentos (MGM). En lo que respecta a la identificación y el análisis de los instrumentos, se usa el estadístico desarrollado por Hansen (1982) (véanse Hall, 2005; Baum, Schaffer y Stillman, 2003, y Hayashi, 2000). A continuación, se muestra la metodología de las VI que se emplea en este estudio para hacer las estimaciones de la FPE.

\section{Resultados}

\section{Análisis de endogeneidad}

La hipótesis nula que se plantea para detectar este problema es $H_{0}: \operatorname{cov}\left(X, \varepsilon_{i}\right)=0$ (insumos exógenos de la FPE). Si el valor $p$ asociado al estadístico MGM es menor que el nivel de significancia, entonces no hay suficiente evidencia estadística para aceptar la hipótesis nula. Por lo tanto, estaríamos ante la presencia de endogeneidad. Al aplicar el estadístico a cada insumo, se encuentra que, al uno por ciento de significancia, la condición de no repetidor es el único factor correlacionado con el error. El resto de las variables no presenta problemas de endogeneidad. Esto se observa para cada una de las competencias evaluadas en PISA 2012.

Por ello, se procede a instrumentalizar el modelo con los instrumentos que se indican a continuación. Primero, la motivación del estudiante (motivacion), medida a través de la respuesta que este da a la siguiente pregunta: "En las últimas dos semanas de clases, ¿cuántas veces se escapó del colegio un día entero?". Si la respuesta es "ninguna vez", la variable toma el valor de 1 y, en caso contrario, toma el valor de 0 . Segundo, los minutos promedio de duración de las clases (minuesp, minumat y minucie) y, tercero, las horas de clase de refuerzo que el estudiante toma fuera del centro escolar (clasesp, clasmat y clascie). Particularmente, en el caso de lectura, los instrumentos empleados son motivacion, minuesp y clasesp; respecto a matemáticas, motivacion, minumat y clasmat, y, en cuanto a ciencias, motivación, minucie y clascie ${ }^{6}$.

\footnotetext{
${ }^{6}$ clasesp, clasmat y clascie son variables dicotómicas que toman el valor de 1, si el estudiante tiene entre dos y cuatro horas de clases de lectura, matemáticas o ciencias fuera del centro escolar, y de 0 , en caso contrario.
} 
Para validar los instrumentos, se plantea la hipótesis sobre su incorrelación (y la de los demás insumos) con el error. Posteriormente, se aplica la prueba de Hansen (1982). Como resultado, se obtiene que el valor $\mathrm{p}$ asociado a este estadístico es mayor que el nivel de significancia del uno por cien en cada área, por lo que no hay suficiente evidencia estadística para rechazar la hipótesis. De esta manera, se concluye que los insumos y los instrumentos son exógenos (véase el cuadro 3).

\section{Cuadro 3}

América Latina (8 países): endogeneidad y sobreidentificación de la función de producción educativa, 2012

\begin{tabular}{lcccccccc}
\hline Prueba de endogeneidad & Argentina & Brasil & Chile & Colombia & Costa Rica & México & Perú & Uruguay \\
\hline Ho: insumos exógenos & & & & & & & & \\
\hline MGM - estadístico chi2 & 72,175 & 82,762 & 60,005 & 8,721 & 27,398 & 290,462 & 56,215 & 9,721 \\
\hline [Lectura] & $(0,000)$ & $(0,000)$ & $(0,000)$ & $(0,000)$ & $(0,000)$ & $(0,000)$ & $(0,000)$ & $(0,002)$ \\
\hline MGM - estadístico chi2 & 71,061 & 5,586 & 26,886 & 8,311 & 7,973 & 353,245 & 90,597 & 11,175 \\
\hline [Matemáticas] & $(0,000)$ & $(0,018)$ & $(0,000)$ & $(0,004)$ & $(0,005)$ & $(0,000)$ & $(0,000)$ & $(0,001)$ \\
\hline MGM - estadístico chi2 & 104,694 & 89,051 & 40,532 & 22,027 & 26,249 & 294,152 & 54,181 & 3,218 \\
\hline [Ciencias] & $(0,000)$ & $(0,000)$ & $(0,000)$ & $(0,000)$ & $(0,000)$ & $(0,000)$ & $(0,000)$ & $(0,073)$ \\
\hline Prueba de instrumentos & & & & & & & & \\
\hline J de Hansen - chi2 & 3,915 & 0,357 & 3,361 & 1,419 & 0,993 & 0,504 & 1,220 & 0,022 \\
\hline [Lectura] & $(0,141)$ & $(0,550)$ & $(0,186)$ & $(0,492)$ & $(0,609)$ & $(0,478)$ & $(0,269)$ & $(0,882)$ \\
\hline J de Hansen - chi2 & 5,337 & 3,484 & 2,000 & 0,590 & 7,554 & 0,708 & 0,374 & 0,016 \\
\hline [Matemáticas] & $(0,069)$ & $(0,062)$ & $(0,157)$ & $(0,745)$ & $(0,023)$ & $(0,400)$ & $(0,541)$ & $(0,899)$ \\
\hline J de Hansen - chi2 & 2,311 & 2,291 & 3,753 & 1,188 & 4,312 & 0,545 & 1,058 & 1,667 \\
\hline [Ciencias] & $(0,317)$ & $(0,130)$ & $(0,053)$ & $(0,552)$ & $(0,116)$ & $(0,460)$ & $(0,304)$ & $(0,197)$ \\
\hline
\end{tabular}

Fuente: Elaboración propia, sobre la base de datos de la Organización de Cooperación y Desarrollo Económicos (OCDE) sobre la prueba PISA 2012.

Nota: Variable instrumentalizada: norepetidor. Instrumentos incluidos: sexo, esfuerzo, disciplina, libros, educamadre, educapadre, emppadre, publico, STRATIO, SCMATEDU, autonomia y SCHSIZE. Instrumentos excluidos: motivacion, minuesp, clasesp y prescolar, que toma el valor de 1 si el estudiante realizó preescolar, 0 si no. Imputación de los datos omitidos a través de la metodología hot-deck según Medina y Galván (2007). Valor p entre paréntesis.

Así, una vez corregido el problema de la endogeneidad (encontrando instrumentos correlacionados con la condición de no repetidor, pero incorrelacionados con el error del modelo), se realizan las estimaciones de la FPE para tratar de identificar los determinantes en la adquisición de competencias en los países de América Latina participantes en el PISA 2012. A continuación, se ofrecen los resultados de cada área, así como una interpretación de los mismos. Como medida de robustez, se presentan al final del artículo los resultados del análisis con datos de PISA 2009. Cabe destacar que los resultados obtenidos fueron similares a los encontrados para 2012 (véase el anexo A1).

\section{Estimación de la función de producción educativa}

\section{a) Comprensión lectora}

En el cuadro 4 se muestran los resultados obtenidos en lectura.

En cuanto a las características individuales, los resultados obtenidos en lectura dan muestra de que existen brechas escolares por sexo en favor de las mujeres, lo que coincide con lo obtenido por Woessmann (2010). Así pues, en el Uruguay y Costa Rica, los países donde la brecha es mayor, las mujeres están 22,0 y 14,1 puntos por encima de los hombres, respectivamente. En la Argentina, el Brasil, Colombia y el Perú, por otro lado, las brechas no son significativas. La condición de no repetidor de curso resulta también un factor determinante en la adquisición de esta competencia en todos los países, pues repercute de forma positiva en la puntuación media, tal y como obtienen Méndez y Zerpa (2011), y Martin (2011). Cabe destacar que el efecto de la variable norepetidor es mayor en 
la Argentina y el Brasil. Lo mismo ocurre con los estudiantes que buscan información adicional para aclarar un tema que estudian y no entienden (variable esfuerzo). En la Argentina y México, el efecto de esta variable es el mayor entre los ocho países. Por último, la disciplina es un condicionante positivo y significativo en Colombia, México y el Uruguay, pero no es significativo en el resto de los países.

Cuadro 4

América Latina (8 países): condicionantes de la adquisición de competencias en lectura, PISA 2012

\begin{tabular}{|c|c|c|c|c|c|c|c|c|c|}
\hline & Var. ind. $\downarrow$ & Argentina & Brasil & Chile & Colombia & Costa Rica & México & Perú & Uruguay \\
\hline \multirow{10}{*}{ 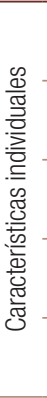 } & \multirow{2}{*}{ constante } & 187,79 & 289,97 & 266,02 & 320,72 & 344,40 & 222,56 & 256,58 & 331,23 \\
\hline & & $(24,995)^{*}$ & $(17,412)^{\star}$ & $(19,678)^{\star}$ & $(30,813)^{\star}$ & $(15,251)^{\star}$ & $(13,521)^{\star}$ & $(15,222)^{*}$ & $(10,379)^{\star}$ \\
\hline & \multirow{2}{*}{ norepetidor } & 268,44 & 230,79 & 210,21 & 155,43 & 129,51 & 227,03 & 180,24 & 134,17 \\
\hline & & $(39,553)^{*}$ & $(30,952)^{*}$ & $(28,176)^{*}$ & $(43,896)^{\star}$ & $(18,069)^{*}$ & $(15,489)^{\star}$ & $(21,903)^{\star}$ & $(20,337)^{\star}$ \\
\hline & \multirow{2}{*}{ sexo } & 7,38 & 6,61 & 9,11 & 1,36 & 14,17 & 5,57 & 3,87 & 22,01 \\
\hline & & $(5,533)$ & $(4,087)$ & $(3,158)^{*}$ & $(5,419)$ & $(3,007)^{\star}$ & $(1,949)^{\star}$ & $(2,877)$ & $(2,758)^{*}$ \\
\hline & \multirow{2}{*}{ esfuerzo } & 10,45 & $-1,17$ & 6,26 & 7,35 & 5,35 & 10,15 & 5,86 & 7,21 \\
\hline & & $(4,493)^{\star \star}$ & $(3,096)$ & $(2,805)^{\star \star}$ & $(2,987)^{\star \star}$ & $(2,389)^{\star \star}$ & $(1,759)^{\star}$ & $(3,046)^{\star \star \star}$ & $(2,428)^{\star}$ \\
\hline & \multirow{2}{*}{ disciplina } & $-11,10$ & 0,29 & 5,48 & 8,59 & 3,99 & 4,44 & $-1,05$ & 6,66 \\
\hline & & $(6,948)$ & $(3,166)$ & $(3,437)$ & $(3,810)^{\star \star}$ & $(2,927)$ & $(2,021)^{\star \star}$ & $(3,631)$ & $(3,108)^{\star \star}$ \\
\hline \multirow{8}{*}{ 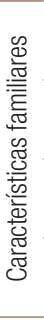 } & \multirow{2}{*}{ libros } & 5,21 & 2,45 & 28,77 & 19,12 & 19,51 & 13,65 & 21,94 & 17,15 \\
\hline & & $(6,090)$ & $(4,130)$ & $(3,140)^{*}$ & $(5,039)^{\star}$ & $(4,089)^{\star}$ & $(2,719)^{*}$ & $(4,185)^{\star}$ & $(3,379)^{\star}$ \\
\hline & \multirow{2}{*}{ educamadre } & 13,97 & 6,45 & 6,93 & 11,03 & 1,21 & 0,07 & 9,64 & 0,97 \\
\hline & & $(4,408)^{*}$ & $(2,805)^{\star \star}$ & $(4,120)$ & $(4,168)^{\star}$ & $(0,321)^{\star}$ & $(1,833)$ & $(3,384)^{*}$ & $(0,531)^{\star \star \star}$ \\
\hline & \multirow{2}{*}{ educapadre } & $-5,20$ & 1,77 & 19,12 & 10,89 & $-2,11$ & 9,31 & 6,26 & $-1,28$ \\
\hline & & $(4,231)$ & $(2,665)$ & $(3,377)^{\star}$ & $(3,157)^{\star}$ & $(2,468)$ & $(1,772)^{\star}$ & $(3,546)^{\star \star \star}$ & $(2,387)$ \\
\hline & \multirow{2}{*}{ emppadre } & 10,84 & 1,94 & $-13,87$ & 2,10 & $-3,41$ & 6,84 & 2,31 & 5,17 \\
\hline & & $(6,636)$ & $(2,897)$ & $(4,691)^{\star}$ & $(4,406)$ & $(3,493)$ & $(2,222)^{*}$ & $(3,478)$ & $(3,583)$ \\
\hline \multirow{10}{*}{  } & \multirow{2}{*}{ publico } & $-16,04$ & $-31,47$ & $-0,84$ & $-27,18$ & $-25,48$ & $-2,55$ & $-25,17$ & $-35,41$ \\
\hline & & $(9,854)$ & $(5,903)^{\star}$ & $(4,736)$ & $(5,543)^{\star}$ & $(4,718)^{\star}$ & $(3,160)$ & $(5,036)^{\star}$ & $(7,505)^{\star}$ \\
\hline & \multirow{2}{*}{ STRATIO } & $-0,08$ & $-0,41$ & $-0,53$ & $-0,37$ & 0,11 & $-0,11$ & $-0,09$ & $-0,16$ \\
\hline & & $(0,135)$ & $(0,068)^{\star}$ & $(0,196)^{*}$ & $(0,152)^{\star \star}$ & $(0,043)^{\star \star}$ & $(0,016)^{\star}$ & $(0,194)$ & $(0,1669)$ \\
\hline & \multirow{2}{*}{ SCMATEDU } & $-9,58$ & $-1,64$ & 0,39 & 3,43 & 3,54 & 3,25 & 10,59 & 9,15 \\
\hline & & $(3,224)^{*}$ & $(1,447)$ & $(1,523)$ & $(1,782)^{\star \star \star}$ & $(1,397)^{\star \star}$ & $(0,893)^{\star}$ & $(1,179)^{\star}$ & $(1,418)^{*}$ \\
\hline & \multirow{2}{*}{ autonomia } & 35,45 & 1,94 & 9,08 & 0,72 & 7,56 & 7,05 & 3,30 & $-2,91$ \\
\hline & & $(8,998)^{*}$ & $(5,818)$ & $(5,418)^{\star \star \star}$ & $(4,265)$ & $(11,100)$ & $(3,185)^{\star \star}$ & $(10,519)$ & $(4,157)$ \\
\hline & \multirow{2}{*}{ SCHSIZE } & $-0,01$ & 0,00 & 0,01 & 0,01 & 0,01 & 0,00 & 0,02 & 0,00 \\
\hline & & $(0,009)$ & $(0,002)$ & $(0,003)^{*}$ & $(0,001)^{\star}$ & $(0,002)^{\star}$ & $(0,001)^{\star \star}$ & $(0,002)^{*}$ & $(0,003)$ \\
\hline & Observaciones & 5632 & 16573 & 5898 & 8059 & 4281 & 29614 & 5442 & 4754 \\
\hline & Instrumentos & $\begin{array}{c}\text { motivacion } \\
\text { minuesp } \\
\text { clasesp }\end{array}$ & $\begin{array}{l}\text { prescolar } \\
\text { minuesp }\end{array}$ & $\begin{array}{l}\text { motivacion } \\
\text { minuesp } \\
\text { clasesp }\end{array}$ & $\begin{array}{l}\text { motivacion } \\
\text { minuesp } \\
\text { clasesp }\end{array}$ & $\begin{array}{l}\text { motivacion } \\
\text { minuesp } \\
\text { clasesp }\end{array}$ & $\begin{array}{l}\text { motivacion } \\
\text { prescolar }\end{array}$ & $\begin{array}{l}\text { motivacion } \\
\text { minuesp }\end{array}$ & $\begin{array}{c}\text { motivacion } \\
\text { minuesp }\end{array}$ \\
\hline
\end{tabular}

Fuente: Elaboración propia, sobre la base de datos de la Organización de Cooperación y Desarrollo Económicos (OCDE) sobre la prueba PISA 2012.

Nota: * significativo al 1\%, ${ }^{* \star}$ significativo al $5 \%$, ${ }^{* \star *}$ significativo al 10\%. Desviaciones estándar robustas entre paréntesis. Imputación de los datos omitidos a través de la metodología hot-deck según Medina y Galván (2007).

Con respecto al estatus socioeconómico y cultural del hogar, los resultados muestran que la cantidad de libros influye de forma positiva en la puntuación media en lectura, tal y como señalaron Crespo, Díaz y Pérez (2012), y Woessmann y otros (2007). El mayor impacto de este indicador se observa en Chile, con 28,7 puntos, y en el Perú, con 21,9 puntos. Sobre el efecto de la educación de la madre, también se observan relaciones positivas, como las que hallaron Hanushek y Luque (2003). Así, los estudiantes con madres cuyo nivel educativo es de al menos bachillerato obtienen una puntuación media mayor que aquellos cuyas madres carecen de estos estudios. En el caso de Chile y México, el efecto de la educación de la madre no es significativo, resultado que coincide con el obtenido por Meunier (2011) en relación con Suiza. Las estimaciones en el caso del nivel educativo del padre 
difieren entre los países. En Chile, Colombia, México y el Perú, la relación es positiva y significativa, mientras que en el resto de los países no lo es. Por último, el efecto del tiempo que dedica el padre al trabajo es muy heterogéneo: negativo, en Chile, positivo, en México, y no significativo en el resto de los países.

Respecto de las características escolares, la relación entre la titularidad del centro escolar y la adquisición de la competencia lectora es negativa en todos los países estudiados. Por tanto, los estudiantes que asisten a colegios privados obtienen una puntuación media mayor que los que asisten a colegios públicos. Sin embargo, en los casos de la Argentina, Chile y México, esta relación no resulta significativa. Formichella (2011) llega a los mismos resultados en cuanto a la Argentina. Respecto a los países en los que esta relación sí que es significativa, el Uruguay y el Brasil son los que presentan mayores diferencias entre los centros públicos y privados, mientras que Costa Rica y el Perú son los que presentan menores diferencias (Giménez y Castro, 2017; Fernández y Del Valle, 2013; Gamboa y Waltenberg, 2012; Montero y otros, 2012)

El coeficiente de la variable que representa la relación alumnos/profesor es negativo, lo que coincide con lo hallado por Krueger (2003), y Krueger y Whitmore (2001). En los casos de la Argentina, el Perú y el Uruguay, esta variable no es significativa. Los valores hallados con respecto al tamaño del centro escolar no fueron significativos en la Argentina, el Brasil y el Uruguay.

La calidad de los materiales educativos también es un condicionante del desempeño escolar, excepto en el Brasil y Chile, donde la relación estimada no es significativa. Por su parte, el Perú y el Uruguay son los países donde se observa el mayor efecto de este tipo de gasto.

Finalmente, respecto al nivel de autonomía, se observa que las decisiones que toman los directores y profesores del centro sobre todas las instancias de peso de la institución tienen un efecto positivo en la Argentina, Chile y México, mientras que dicho efecto no es significativo en el resto de los países (Benton, 2014).

\section{b) Matemáticas}

En el cuadro 5 se muestran los factores que contribuyen a explicar los resultados en matemáticas.

Respecto a las diferencias por sexo, se observa que en todos los países objeto de nuestra investigación, los hombres obtuvieron una puntuación media superior a la de las mujeres en matemáticas, resultados similares a los obtenidos por Vegas y Petrow (2007). Cabe destacar que la mayor brecha se da en la Argentina, Colombia y el Perú. Por su parte, el efecto de la variable norepetidor es positivo y significativo. Esta estimación coincide con lo encontrado por Oreiro y Valenzuela (2013), y Méndez y Zerpa (2011). Los estudiantes con mayor capacidad académica (mayor efecto de la variable norepetidor) son los de México y la Argentina. El esfuerzo, por su parte, tiene un efecto diferencial positivo y significativo, excepto en los estudiantes peruanos. En cuanto al clima disciplinario, se observa una relación positiva y significativa en el Brasil, Colombia, México y el Uruguay. Así, los estudiantes que prestan atención al profesor logran una puntuación media que es $3,7,7,5,6,2$ y 6,6 puntos superior a la de los que no, respectivamente. El efecto no es significativo en la Argentina, el Perú, Chile y Costa Rica.

En cuanto a las características familiares, la cantidad de libros es un factor condicionante de la adquisición de competencias. El efecto es mayor en Chile y el Perú. La brecha por nivel educativo de la madre en general es positiva y significativa, al igual que en Hanushek y Luque (2003). Ello determina una mayor puntuación media para los estudiantes cuyas madres terminaron al menos el bachillerato. Los países donde la educación de la madre tiene un mayor impacto son Chile y el Brasil. Sin embargo, en el Perú, el efecto de la educación de la madre no es significativo, al igual que ocurre en México. 


\section{Cuadro 5}

América Latina (8 países): condicionantes de la adquisición de competencias en matemáticas, 2012

\begin{tabular}{|c|c|c|c|c|c|c|c|c|c|}
\hline & Var. ind. $\downarrow$ & Argentina & Brasil & Chile & Colombia & Costa Rica & México & Perú & Uruguay \\
\hline \multirow{10}{*}{ 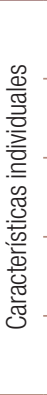 } & \multirow{2}{*}{ constante } & 243,70 & 375,54 & 294,16 & 349,90 & 374,86 & 229,23 & 273,57 & 357,42 \\
\hline & & $(20,223)^{\star}$ & $(11,971)^{\star}$ & $(17,557)^{\star}$ & $(22,527)^{\star}$ & $(12,773)^{\star}$ & $(14,124)^{\star}$ & $(16,109)^{\star}$ & $(9,347)^{\star}$ \\
\hline & \multirow{2}{*}{ norepetidor } & 221,31 & 101,05 & 161,01 & 121,02 & 88,22 & 239,09 & 193,38 & 130,63 \\
\hline & & $(32,461)^{*}$ & $(21,946)^{\star}$ & $(24,873)^{*}$ & $(31,947)^{\star}$ & $(15,222)^{*}$ & $(16,106)^{*}$ & $(23,282)^{\star}$ & $(17,966)^{*}$ \\
\hline & \multirow{2}{*}{ sexo } & $-38,68$ & $-27,01$ & $-34,91$ & $-38,63$ & $-30,27$ & $-33,31$ & $-36,06$ & $-23,21$ \\
\hline & & $(4,589)^{\star}$ & $(2,801)^{\star}$ & $(2,793)^{\star}$ & $(4,111)^{\star}$ & $(2,441)^{\star}$ & $(1,993)^{\star}$ & $(2,972)^{\star}$ & $(2,489)^{*}$ \\
\hline & \multirow{2}{*}{ esfuerzo } & 10,84 & 6,12 & 7,75 & 6,52 & 5,37 & 9,33 & 3,98 & 9,45 \\
\hline & & $(3,665)^{\star}$ & $(2,009)^{\star}$ & $(2,439)^{\star}$ & $(2,525)^{\star}$ & $(1,947)^{\star}$ & $(1,789)^{*}$ & $(3,155)$ & $(2,284)^{*}$ \\
\hline & \multirow{2}{*}{ disciplina } & $-6,95$ & 3,78 & 2,28 & 7,53 & 2,75 & 6,24 & $-2,31$ & 6,68 \\
\hline & & $(5,640)$ & $(1,983)^{\star \star \star}$ & $(2,936)$ & $(3,028)^{\star \star}$ & $(2,489)$ & $(2,051)^{\star}$ & $(3,668)$ & $(2,839)^{\star \star}$ \\
\hline \multirow{8}{*}{ 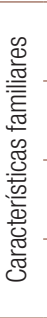 } & \multirow{2}{*}{ libros } & 9,70 & 10,84 & 34,63 & 21,61 & 24,72 & 16,12 & 27,43 & 23,12 \\
\hline & & $(5,078)^{\star \star \star}$ & $(2,832)^{\star}$ & $(2,851)^{\star}$ & $(4,342)^{\star}$ & $(3,833)^{\star}$ & $(2,740)^{*}$ & $(4,405)^{\star}$ & $(3,062)^{\star}$ \\
\hline & \multirow{2}{*}{ educamadre } & 8,06 & 10,74 & 11,30 & 10,09 & 0,96 & $-1,17$ & 4,83 & 1,41 \\
\hline & & $(3,614)^{\star \star}$ & $(1,774)^{\star}$ & $(3,441)^{\star}$ & $(3,404)^{\star}$ & $(0,261)^{\star}$ & $(1,876)$ & $(3,511)$ & $(0,479)^{\star}$ \\
\hline & \multirow{2}{*}{ educapadre } & 1,95 & 6,66 & 20,49 & 9,00 & 0,42 & 6,69 & 3,04 & $-2,13$ \\
\hline & & $(3,475)$ & $(1,666)^{\star}$ & $(2,833)^{\star}$ & $(2,576)^{\star}$ & $(1,972)$ & $(1,814)^{\star}$ & $(3,711)$ & $(2,192)$ \\
\hline & \multirow{2}{*}{ emppadre } & 8,91 & $-0,04$ & $-6,03$ & 0,54 & $-1,37$ & 0,65 & $-0,98$ & 5,34 \\
\hline & & $(5,418)$ & $(1,754)$ & $(3,930)$ & $(3,538)$ & $(2,797)$ & $(2,307)$ & $(3,607)$ & $(3,145)^{\star \star \star}$ \\
\hline \multirow{10}{*}{  } & \multirow{2}{*}{ publico } & $-8,66$ & $-51,66$ & $-8,80$ & $-24,03$ & $-28,04$ & $-0,35$ & $-25,88$ & $-30,01$ \\
\hline & & $(8,046)$ & $(3,956)^{\star}$ & $(4,092)^{\star *}$ & $(4,557)^{\star}$ & $(4,200)^{\star}$ & $(3,257)$ & $(5,435)^{\star}$ & $(6,686)^{\star}$ \\
\hline & \multirow{2}{*}{ STRATIO } & $-0,10$ & $-0,45$ & $-0,76$ & $-0,59$ & 0,17 & $-0,10$ & $-0,11$ & $-0,64$ \\
\hline & & $(0,107)$ & $(0,046)^{\star}$ & $(0,163)^{\star}$ & $(0,127)^{\star}$ & $(0,044)^{\star}$ & $(0,017)^{\star}$ & $(0,205)$ & $(0,170)^{\star}$ \\
\hline & \multirow{2}{*}{ SCMATEDU } & $-7,88$ & 3,61 & 1,37 & 3,30 & 6,64 & 1,47 & 9,15 & 7,13 \\
\hline & & $(2,628)^{*}$ & $(0,956)^{\star}$ & $(1,300)$ & $(1,403)^{\star \star}$ & $(1,142)^{\star}$ & $(0,910)$ & $(1,229)^{\star}$ & $(1,287)^{\star}$ \\
\hline & \multirow{2}{*}{ autonomia } & 22,47 & 2,70 & 14,19 & $-4,29$ & $-1,36$ & 5,55 & $-6,39$ & $-5,18$ \\
\hline & & $(7,158)^{*}$ & $(3,432)$ & $(4,897)^{\star}$ & $(3,456)$ & $(9,592)$ & $(3,227)^{\star \star \star}$ & $(11,135)$ & $(3,778)$ \\
\hline & \multirow{2}{*}{ SCHSIZE } & $-0,02$ & 0,01 & 0,01 & 0,01 & 0,01 & 0,00 & 0,01 & 0,00 \\
\hline & & $(0,008)^{\star \star}$ & $(0,002)^{\star}$ & $(0,003)^{\star}$ & $(0,001)^{\star}$ & $(0,002)^{\star}$ & $(0,001)$ & $(0,003)^{\star}$ & $(0,003)$ \\
\hline & Observaciones & 5632 & 16968 & 5898 & 8059 & 4281 & 29614 & 5442 & 4754 \\
\hline & Instrumentos & $\begin{array}{c}\text { motivacion } \\
\text { minumat }\end{array}$ & $\begin{array}{c}\text { motivacion } \\
\text { minumat } \\
\text { clasmat }\end{array}$ & $\begin{array}{c}\text { motivacion } \\
\text { minumat } \\
\text { clasmat }\end{array}$ & $\begin{array}{c}\text { motivacion } \\
\text { minumat } \\
\text { clasmat }\end{array}$ & $\begin{array}{c}\text { motivacion } \\
\text { minumat } \\
\text { clasmat }\end{array}$ & $\begin{array}{c}\text { motivacion } \\
\text { prescolar }\end{array}$ & $\begin{array}{c}\text { motivacion } \\
\text { minumat }\end{array}$ & $\begin{array}{c}\text { motivacion } \\
\text { minumat }\end{array}$ \\
\hline
\end{tabular}

Fuente: Elaboración propia, sobre la base de datos de la Organización de Cooperación y Desarrollo Económicos (OCDE) sobre la prueba PISA 2012

Nota: * significativo al 1\%, ${ }^{\star \star}$ significativo al 5\%, ${ }^{\star \star \star}$ significativo al 10\%. Desviaciones estándar robustas entre paréntesis. Imputación de los datos faltantes a través de la metodología hot-deck, según Medina y Galván (2007).

El efecto del nivel educativo del padre solo es positivo y significativo en Chile, Colombia, el Brasil y México. De esta manera, los estudiantes cuyos padres tienen al menos el bachillerato logran, respectivamente, 20,4, 9,0, 6,6 y 6,6 puntos más que aquellos cuyo padre no ha alcanzado este nivel educativo. En lo que respecta a la Argentina, Costa Rica y el Perú, se calcula una relación positiva pero no significativa. En el caso del Uruguay, dicha relación es negativa y no significativa. Por último, el tipo de contrato no resulta un condicionante de la adquisición de esta competencia, ya que los coeficientes estimados no son significativos, excepto en el Uruguay.

En cuanto a las variables relacionadas con la escuela, se aprecian brechas significativas entre el desempeño de los colegios públicos y los privados, con la excepción de la Argentina y México. En el Brasil y el Uruguay se observan las mayores divergencias, como ocurre con compresión lectora. Entre tanto, en Chile y Colombia estas diferencias son menores. 
El tamaño de la clase tiene un efecto negativo y significativo (menos en Costa Rica). El tamaño del centro escolar afecta positiva y significativamente en todos los países, menos en la Argentina (donde es negativo y significativo) y en México y el Uruguay (no significativo).

Respecto al efecto de la autonomía, los resultados son ambiguos. En el caso de la Argentina, Chile y México, el que las decisiones sobre las instancias importantes de la institución estén a cargo del director y los profesores del centro favorece el desempeño de los estudiantes en matemáticas. En promedio, en estos países, los centros donde hay autonomía logran una puntuación 22,4 14,1 y 5,5 puntos mayor que la de los centros donde no la hay. En el Brasil, Colombia, Costa Rica, el Perú y el Uruguay, la relación no es significativa.

Finalmente, la calidad de los bienes educativos guarda una relación positiva con el desempeño en matemáticas. Los países donde hay un mayor efecto son el Perú, el Uruguay y Costa Rica. Cabe destacar que la Argentina es un caso especial, ya que el impacto del gasto educativo sobre el desempeño es negativo.

\section{c) Ciencias}

En el caso de ciencias, como principales condicionantes de la adquisición de esta competencia se encuentran entre las características individuales, el sexo y la condición de no repetidor (véase el cuadro 6). En cuanto al primer factor, se estimaron brechas negativas y significativas, al igual que en Vegas y Petrow (2007). Las mujeres obtuvieron una menor puntuación media. Respecto del segundo, la relación entre la no repetición y el desempeño académico es positiva y significativa. Los estudiantes que no repiten el curso logran un mejor desempeño que los que sí lo han hecho al menos una vez (véanse Oreiro y Valenzuela, 2013, y Méndez y Zerpa, 2011).

\section{Cuadro 6}

América Latina: condicionantes de la adquisición de competencias en ciencias, 2012

\begin{tabular}{|c|c|c|c|c|c|c|c|c|c|}
\hline & Var. ind. $\downarrow$ & Argentina & Brasil & Chile & Colombia & Costa Rica & México & Perú & Uruguay \\
\hline \multirow{10}{*}{ 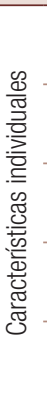 } & \multirow{2}{*}{ constante } & 193,34 & 403,52 & 309,39 & 298,60 & 365,93 & 249,65 & 286,18 & 374,63 \\
\hline & & $(27,767)^{\star}$ & $(11,480)^{\star}$ & $(15,814)^{\star}$ & $(35,901)^{\star}$ & $(15,541)^{\star}$ & $(12,699)^{\star}$ & $(12,842)^{\star}$ & $(10,428)^{\star}$ \\
\hline & \multirow{2}{*}{ norepetidor } & 299,20 & 51,37 & 158,77 & 214,08 & 123,92 & 203,04 & 145,94 & 109,86 \\
\hline & & $(44,942)^{\star}$ & $(21,133)^{\star \star}$ & $(21,786)^{\star}$ & $(51,400)^{\star}$ & $(18,022)^{\star}$ & $(14,451)^{\star}$ & $(18,886)^{\star}$ & $(19,980)^{\star}$ \\
\hline & \multirow[b]{2}{*}{ sexo } & $-26,43$ & $-5,17$ & $-16,58$ & $-40,42$ & $-22,28$ & $-22,71$ & $-19,28$ & $-9,80$ \\
\hline & & $(6,118)^{*}$ & $(2,658)^{\star \star \star}$ & $(2,731)^{\star}$ & $(6,354)^{*}$ & $(2,870)^{\star}$ & $(1,767)^{\star}$ & $(2,473)^{\star}$ & $(2,673)^{\star}$ \\
\hline & & 8,06 & 8,76 & 7,21 & 3,61 & 3,23 & 8,34 & 4,51 & 10,27 \\
\hline & & $(4,855)^{\star \star \star}$ & $(1,914)^{\star}$ & $(2,520)^{*}$ & $(3,583)$ & $(2,236)$ & $(1,592)^{\star}$ & $(2,599)^{\star \star \star}$ & $(2,407)^{\star}$ \\
\hline & \multirow{2}{*}{ disciplina } & $-11,50$ & 6,89 & 4,67 & 5,40 & $-0,58$ & 3,15 & $-1,63$ & 4,12 \\
\hline & & $(7,742)$ & $(1,907)^{\star}$ & $(3,120)$ & $(4,402)$ & $(2,804)$ & $(1,835)^{\star \star \star}$ & $(3,079)$ & $(3,080)$ \\
\hline \multirow{8}{*}{ 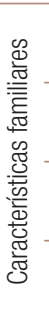 } & \multirow[b]{2}{*}{ libros } & 3,51 & 10,39 & 28,47 & 18,05 & 17,48 & 15,52 & 27,31 & 21,59 \\
\hline & & $(6,687)$ & $(2,896)^{\star}$ & $(2,967)^{\star}$ & $(5,916)^{\star}$ & $(3,712)^{\star}$ & $(2,469)^{\star}$ & $(3,678)^{*}$ & $(3,375)^{\star}$ \\
\hline & \multirow{2}{*}{ educamadre } & 11,16 & 12,46 & 8,10 & 0,87 & 0,99 & 0,44 & 8,73 & 1,92 \\
\hline & & $(4,834)^{\star \star}$ & $(1,738)^{\star}$ & $(3,450)^{\star \star}$ & $(4,952)$ & $(0,315)^{\star}$ & $(1,680)$ & $(2,886)^{*}$ & $(0,507)^{\star}$ \\
\hline & \multirow{2}{*}{ educapadre } & 2,21 & 8,71 & 19,30 & 10,89 & $-2,94$ & 8,37 & 3,66 & 0,41 \\
\hline & & $(4,633)$ & $(1,626)^{*}$ & $(2,998)^{\star}$ & $(3,731)^{\star}$ & $(2,352)$ & $(1,630)^{*}$ & $(3,050)$ & $(2,321)$ \\
\hline & \multirow{2}{*}{ emppadre } & 9,99 & 1,02 & $-7,76$ & 2,69 & $-1,06$ & 2,27 & $-2,24$ & $-0,70$ \\
\hline & & $(7,234)$ & $(1,705)$ & $(4,042)^{\star \star \star}$ & $(5,279)$ & $(3,276)$ & $(2,012)$ & $(3,056)$ & $(3,401)$ \\
\hline
\end{tabular}


Cuadro 6 (conclusión)



Fuente: Elaboración propia, sobre la base de datos de la Organización de Cooperación y Desarrollo Económicos (OCDE) sobre la prueba PISA 2012.

Nota: * significativo al 1\%, ${ }^{* \star}$ significativo al 5\%, ${ }^{* \star}$ significativo al 10\%. Desviaciones estándar robustas entre paréntesis. Imputación de los datos faltantes a través de la metodología hot-deck según Medina y Galván (2007).

Entre las características socioeconómicas y culturales del estudiante, los principales condicionantes son la cantidad de libros en el hogar (Woessmann y otros, 2007) y la educación de la madre (Hanushek y Luque, 2003). En lo que respecta al primer factor, la Argentina es un caso excepcional y, en cuanto al segundo, lo son Colombia y México, ya que los coeficientes asociados a estas variables no resultaron significativos. Con respecto al efecto de la educación del padre, se observa que, si bien el efecto estimado sobre la adquisición de competencias en ciencias es positivo (menos en Costa Rica), solo resulta significativo en el Brasil, Chile, Colombia y México. Ahora bien, los resultados del tipo de contrato del padre son más heterogéneos. En unos casos, el efecto es negativo y no significativo (Costa Rica, Perú y Uruguay), en otros, negativo y significativo (Chile) y, en otros, positivo y no significativo (Argentina, Brasil, Colombia y México).

En cuanto a los aspectos escolares, en esta área también se estimaron brechas medias en el desempeño escolar entre los colegios públicos y privados, y esas brechas favorecen a estos últimos. No obstante, en el caso de la Argentina, Colombia y México, las brechas no son significativas. El Brasil y el Uruguay son los países con la mayor diferencia, como ocurre en lectura y matemáticas. Respecto de la proporción de alumnos por profesor, se obtienen resultados similares a los encontrados en lectura y matemáticas. El efecto sobre el rendimiento escolar es negativo y significativo, si bien no es significativo en los casos de Argentina, Costa Rica, el Perú y el Uruguay. El tamaño del centro escolar tiene un impacto positivo y significativo, excepto en los casos de México y el Uruguay.

En cuanto a la autonomía del centro, se estiman efectos positivos y significativos en la Argentina, el Brasil, Chile y México. El efecto es negativo y significativo en el Uruguay, y no es significativo en Colombia, Costa Rica y el Perú.

Por último, en el caso de la calidad de los materiales educativos, los coeficientes son positivos y estadísticamente significativos en todos los países, menos en Argentina, que es negativo y significativo, y en Colombia, que es no significativo. 


\section{d) Descomposición de las causas de las diferencias en el rendimiento}

Las puntuaciones desiguales obtenidas por los alumnos se pueden deber a diferencias en el esfuerzo que realizan, lo que corresponde a la parte residual de la FPE, o a factores que escapan a su control, es decir, a insumos definidos en dicha función: factores individuales, familiares y escolares.

Sea $r$ el resultado obtenido en las pruebas PISA y $C$ una matriz de factores no controlables. El resultado esperado de las pruebas, condicionado a estos factores, será el siguiente:

$$
\hat{r}=\mathrm{E}[r \mid C]
$$

A partir de (2), podemos descomponer la varianza de los resultados en las distintas áreas, $\frac{1}{N} \sum_{i=1}^{N}\left(r_{i}-\bar{r}\right)^{2}$, en función de la contribución relativa de cada factor explicativo incluido en la FPE. Para ello, utilizamos la metodología de Shapley-Shorrocks ${ }^{7}$. El método se basa en el cálculo de la varianza, considerando todas las permutaciones posibles de las variables explicativas recogidas en la FPE. Como los resultados escolares en PISA son variables continuas de media y varianza arbitraria, la mejor opción para estimar el modelo (2) será usar una función lineal (Ferreira y Gignoux, 2014).

El cuadro 7 contiene la descomposición de Shapley-Shorrocks en función de las características del estudiante, del hogar y de la escuela. La estimación de la varianza de los resultados en matemáticas, lengua y ciencias en relación con todas las observaciones de América Latina es de 0,186, 0,185 y 0,181. En lo que respecta a los países, la varianza mínima corresponde a México, 0,141 en matemáticas y ciencias, y la máxima a Chile, 0,373 en matemáticas. La validez de estas estimaciones queda avalada por el bajo valor de los errores estándar del método de remuestreo bootstrap, significativo a los niveles estándar con 100 réplicas.

En el resto del cuadro se muestran los porcentajes de descomposición de la varianza, esto es, en qué medida el éxito escolar está condicionado por las características individuales, familiares y escolares. En promedio, las características individuales explicarían el 12\% de la variación de los resultados escolares, las familiares, el 28\%, y las escolares, el 60\%. Cabe destacar que las características individuales juegan un papel especialmente relevante en Colombia y México, las familiares, en Chile, Colombia y el Perú, y las escolares, en la Argentina, el Brasil y Costa Rica.

7 Véase una explicación pormenorizada en Shorrocks (1982). 


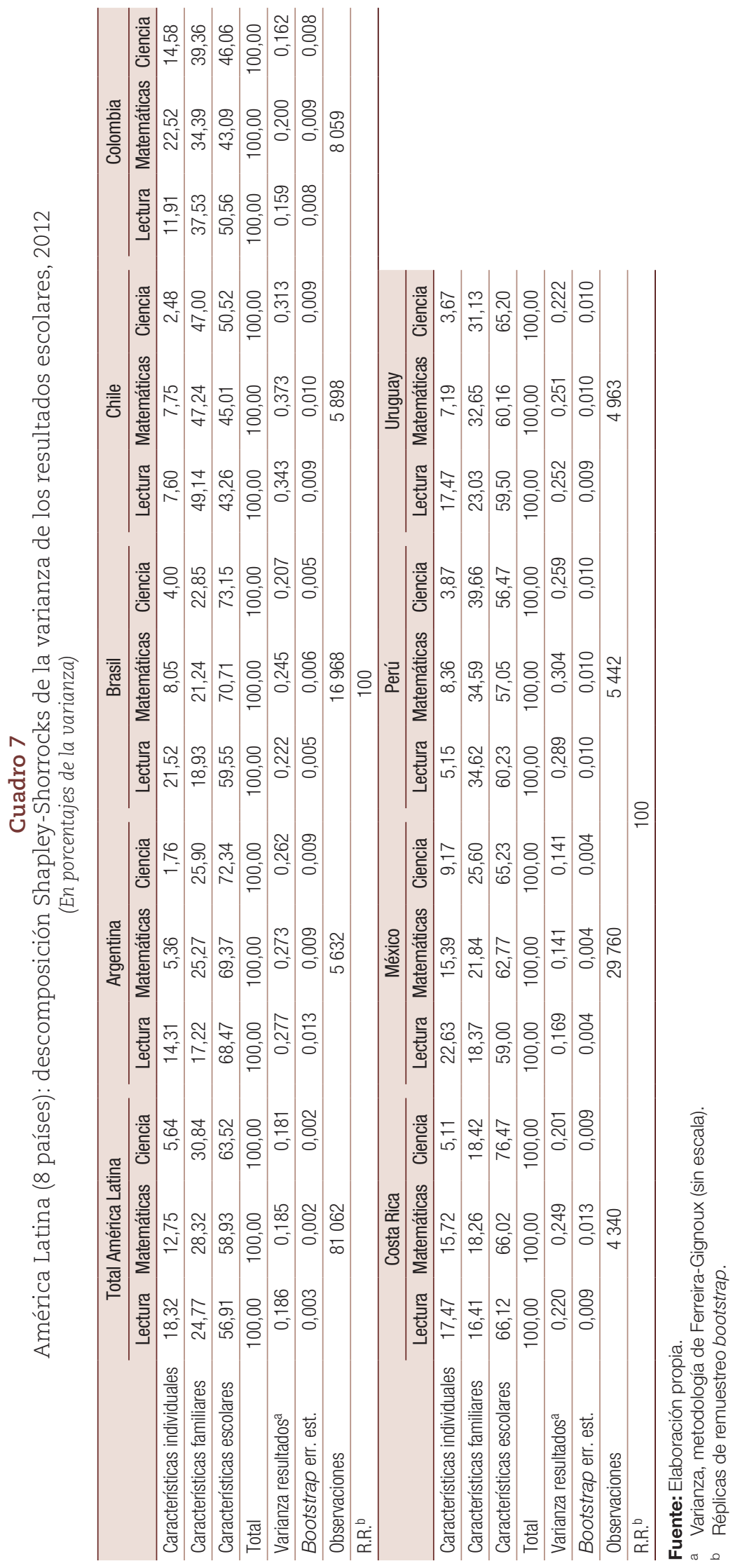




\section{Conclusiones}

En este trabajo se identifican los factores determinantes del rendimiento académico en los países de América Latina que participaron en PISA 2012. Al trabajar con funciones de producción educativas, la existencia de problemas de endogeneidad conlleva que los coeficientes estimados sean inconsistentes y sesgados. Por este motivo, las estimaciones a través de modelos multinivel no son óptimas. La aportación metodológica de este trabajo reside en la utilización de la técnica de las variables instrumentales, que permite corregir los problemas de endogeneidad.

La descomposición de la varianza de los resultados escolares revela que son las características escolares las que explicarían en mayor medida la variabilidad de los resultados entre los alumnos (explicarían el $60 \%$ de esta variabilidad en el conjunto de América Latina). Le siguen las características familiares (28\%) y las individuales (12\%). Las características de las escuelas juegan un papel especialmente relevante en la Argentina, el Brasil y Costa Rica; las familiares, en Chile, Colombia y el Perú, y, las individuales, en Colombia y México. La descomposición para obtener el aporte de cada factor y la constatación de la importancia que tienen los factores correspondientes a la escuela suponen una novedad en los estudios sobre el conjunto de América Latina.

Respecto de los factores individuales y familiares, se estimaron brechas significativas por sexo. En efecto, las mujeres obtuvieron una mayor puntuación media en lectura, mientras que los hombres lo hicieron en matemáticas y ciencias. La retención escolar (no repetidor de curso) resultó ser un factor determinante del desempeño escolar, al igual que la cantidad de libros en el hogar y el nivel educativo de la madre.

En el caso de los factores escolares, los efectos son más heterogéneos. Asistir a colegio privado tiene un efecto positivo y significativo en el Brasil, Chile, Colombia, Costa Rica, México, el Perú y el Uruguay. Una disminución del número de alumnos por aula tiene un efecto positivo en los centros del Brasil, Chile, Colombia y México. Un mayor gasto en materiales educativos produciría mejores resultados, si bien en la Argentina este efecto no se constata. El tamaño de los centros influye positivamente en los resultados, excepto en la Argentina y el Uruguay. Y, por último, el que haya más autonomía en la gestión de los centros afecta positivamente los resultados de los estudiantes de la Argentina, Chile y México.

La constatación de que existen problemas de endogeneidad dentro de la función de producción educativa de los países de América Latina nos lleva a aconsejar la utilización de técnicas consistentes y robustas ante estos problemas. Frente a otras técnicas que son habituales en otros trabajos, pero en las que no se tiene en cuenta la endogeneidad entre las variables del modelo, el uso de variables instrumentales permitirá aproximarnos al verdadero efecto de los insumos educativos sobre los resultados escolares. 


\section{Bibliografía}

Alexander, L. y J. Simmons (1975), "The determinants of school achievement in developing countries: the educational production function”, Working Paper, № 201, Washington, D.C., Banco Mundial.

Angrist, J. D. (2014), "The perils of peer effects", Labour Economics, vol. 30, Amsterdam, Elsevier.

Ayala, J., S. Marrugo y B. Saray (2011), "Antecedentes familiares y rendimiento académico en los colegios oficiales de Cartagena”, Economía y Región, vol. 5, №2, Cartagena de Indias, Universidad Tecnológica de Bolívar.

Banco Mundial (2005), "Mexico: determinants of learning policy note", Informe, № 31842-MX, Washington, D.C. Baum, C. F., M. E. Schaffer y S. Stillman (2003), "Instrumental variables and GMM: estimation and testing", The Stata Journal, vol. 3, № 1, Texas, Stata Corporation.

Benton, T. (2014), A Re-evaluation of the Link Between Autonomy, Accountability and Achievement in PISA 2009, Cambridge, Cambridge Assessment.

Bonilla, L. (2011), "Doble jornada escolar y calidad de la educación en Colombia”, Documentos de Trabajo sobre Economía Regional y Urbana, №143, Bogotá, Banco de la República.

Brunello, G. y L. Rocco (2008), "Educational standards in private and public schools", The Economic Journal, vol. 118, No 533, Wiley.

Cárcamo, C. y J. Mola (2012), "Diferencias por sexo en el desempeño académico en Colombia: un análisis regional”, Economía y Región, vol. 6, № 1, Cartagena de Indias, Universidad Tecnológica de Bolívar.

Cervini, R. (2012), "El 'efecto escuela' en países de América Latina: reanalizando los datos del SERCE", Archivos Analiticos de Politicas Educativas, vol. 20, №39.

(2003), "Diferencias de resultados cognitivos y no-cognitivos entre estudiantes de escuelas públicas y privadas en la educación secundaria de Argentina: un análisis multinivel", Archivos Analíticos de Políticas Educativas, vol. 11, № 6.

CIPPEC (Centro de Implementación de Políticas para la Equidad y el Crecimiento) (2011) [en línea] http://www.cippec.org/publicaciones-educacion.

Coleman, J. y otros (1966), Equality of Educational Opportunity, Washington, D.C., Departamento de Educación.

Crespo, E., C. Díaz y J. Pérez (2012), "Factores condicionantes de la desigualdad educativa: un análisis para el caso español", Investigaciones de economía de la educación, E. Pacheco Vieira (ed.), vol. 7, Oporto, Asociación de Economía de la Educación.

Donoso, S. y G. Hawes (2002), "Eficiencia escolar y diferencias socioeconómicas: a propósito de los resultados de las pruebas de medición de la calidad de la educación en Chile", Educação e Pesquisa, vol. 28, №2, São Paulo, Universidad de São Paulo.

Durrant, G. B. (2009), "Imputation methods for handling item-nonresponse in practice: methodological issues and recent debates", International Journal of Social Research Methods, vol. 12, № 4, Taylor \& Francis.

Fernández, A. y R. del Valle (2013), "Desigualdad educativa en Costa Rica: la brecha entre estudiantes de colegios públicos y privados. Análsis con los resultados de la evaluación internacional PISA", Revista CEPAL, vol. 111 (LC/G.2597-P), Santiago, Comisión Económica para América Latina y el Caribe (CEPAL).

Ferreira, F. y J. Gignoux (2014), "The measurement of educational inequality: achievement and opportunity", World Bank Economic Review, vol. 28, № 2, Washington, D.C., Banco Mundial.

Formichella, M. M. (2011), "¿Se debe el mayor rendimiento de las escuelas de gestión privada en la Argentina al tipo de administración?”, Revista CEPAL, № 105 (LC/G.2508-P), Santiago, Comisión Económica para América Latina y el Caribe (CEPAL).

Gamboa, L. F. y F. D. Waltenberg (2012), "Inequality of opportunity for educational achievement in Latin America: evidence from PISA 2006-2009", Economics of Education Review, vol. 31, № 5, Amsterdam, Elsevier.

García, I., M. Hidalgo y A. Robles (2010), "Diferencias regionales en rendimiento educativo en España: ¿la familia lo explica todo?", Documento de Trabajo, № 10.12, Sevilla, Universidad Pablo de Olavide.

Gaviria, J. L. y M. Castro (2005), Modelos jerárquicos lineales, Madrid, La Muralla.

Giménez, G. y G. Castro (2017), "¿Por qué los estudiantes de colegios públicos y privados de Costa Rica obtienen diferentes resultados académicos?", Perfiles Latinoamericanos, vol. 25, № 49, Ciudad de México, Facultad Latinoamericana de Ciencias Sociales (FLACSO).

Greene, W. H. (2012), Econometric Analysis, New Jersey, Prentice Hall.

Hall, A. R. (2005), Generalized Method of Moments, Oxford, Oxford University Press.

Hansen, L. P. (1982), "Large sample properties of generalized method of moments estimators", Econometrica, vol. 50, N 4, Nueva York, The Econometric Society. 
Hanushek, E. A. (2011), "The economic value of higher teacher quality", Economics of Education Review, vol. 30, N 3, Amsterdam, Elsevier.

Hanushek, E. A., S. Link y L. Woessmann (2013), "Does school autonomy make sense everywhere? Panel estimates from PISA", Journal of Development Economics, vol. 104, Amsterdam, Elsevier.

Hanushek, E. A. y J. Luque (2003), "Efficiency and equity in schools around the world", Economics of Education Review, vol. 22, № 5, Amsterdam, Elsevier.

Hanushek, E. A. y L. Woessmann (2012), "Schooling, educational achievement, and the Latin American growth puzzle", Journal of Development Economics, vol. 99, № 2, Amsterdam, Elsevier. (2011), "The economics of international diferences in educational achievement", Handbook of the Economics of Education, E. A. Hanushek, S. Machin y L. Woessmann (eds.), vol. 3. Amsterdam, North-Holland.

Hayashi, F. (2000), Econometrics, New Jersey, Princeton University.

Hindrinks, J. y otros (2010), "School autonomy and educational performance: within-country evidence", CORE Discussion Papers, № 2010082, Lovaina, Universidad Católica de Lovaina.

Hong, G. y B. Yu (2007), "Early-grade retention and children's reading and math learning in elementary years", Educational Evaluation and Policy Analysis, vol. 29, № 4, SAGE.

Jencks, C. (1972), Inequality: A Reassessment of the Effect of Family and Schooling in America, Nueva York, Basic Books.

Krueger, A. B. (2003), "Economic considerations and class size", The Economic Journal, vol. 113, № 485, Wiley.

Krueger, A. B. y D. M. Whitmore (2001), "The effect of attending a small class in the early grades on collegetest taking and middle school test results: evidence from project STAR", The Economic Journal, vol. 111, $N^{\circ} 468$, Wiley.

León, G., J. Manzi y R. Paredes (2004), Calidad docente y rendimiento escolar en Chile: evaluando la evaluación, Santiago, Pontificia Universidad Católica de Chile.

Marteleto, L. (2012), "Educational inequality by race in Brazil, 1982-2007: structural changes and shifts in racial classification", Demography, vol. 49, № 1, Springer.

Martin, A. (2011), "Holding back and holding behind: grade retention and students' non academic and academic outcomes", British Educational Research Journal, vol. 37, N 5, Taylor \& Francis.

Medina, F. y M. Galván (2007), "Imputación de datos: teoría y práctica", serie Estudios Estadísticos y Prospectivos, № 54 (LC/L.2772-P), Santiago, Comisión Económica para América Latina y el Caribe (CEPAL).

Méndez, N. y M. Zerpa (2011), "Desigualdad en las capacidades educativas. Los casos de Uruguay y Chile", Revista de Economía, vol. 18, № 1, Montevideo, Banco Central del Uruguay.

Meunier, M. (2011), "Immigration and student achievement: evidence from Switzerland", Economics of Education Review, vol. 30, № 1, Amsterdam, Elsevier.

Montero, E. y otros (2012), Costa Rica en la pruebas PISA 2009 de competencia lectora y alfabetización matemática, San José, Consejo Nacional de Rectores (CONARE).

Mullis, I. V. S. y otros (2007), PIRLS 2006 International Report. IEA's Progress in International Reading Literacy Study in Primary Schools in 40 Countries, Chesnut Hill, Massachusetts, Boston College.

Murnane, R. J. (1981), "Interpreting the evidence on school effectiveness", Teachers College Record, vol. $83, N^{\circ} 1$.

OCDE (Organización de Cooperación y Desarrollo Económicos) (2015), "Programme for International Student Assessment" [en línea] http://pisa2012.acer.edu.au/. (2014), PISA 2012 Technical Report, París.

Oreiro, C. y J. P. Valenzuela (2013), "Factores que determinan el desempeño educativo en el Uruguay, 20032006", Revista CEPAL, № 107 (LC/G.2536-P), Santiago, Comisión Económica para América Latina y el Caribe (CEPAL).

Post, D. (2011), "Primary school student employment and academic achievement in Chile, Colombia, Ecuador and Perú", Revista Internacional del Trabajo, vol. 150, №34, Ginebra, Organización Internacional del Trabajo (OIT).

Rivas, A. (2015), América Latina depués de PISA. Lecciones aprendidas de la educación en siete países (20002015), Buenos Aires, Centro de Implementación de Políticas Públicas para la Equidad y el Crecimiento (CIPPEC)/Natura/Instituto Natura.

Sánchez, A. (2011), "Etnia y desempeño académico en Colombia", Documentos de Trabajo sobre Economía Regional, № 156, Cartagena, Banco de la República.

Shorrocks, A. F. (1982), "Inequality decomposition by factor components", Econometrica, vol. 50, № 1, Nueva York, The Econometric Society. 
Thieme, C., D. Prior y E. Tortosa-Ausina (2013), "A multilevel decomposition of school performance using robust nonparametric frontier techniques", Economics of Education Review, № 32, Amsterdam, Elsevier.

Vegas, E. y J. Petrow (2007), Raising Student Achievement in Latin America: The Challenge for the 21st Century, Washington, D.C., Banco Mundial.

Viáfara, C. y F. Urrea (2006), "Efectos de la raza y el género en el logro educativo y estatus socio-ocupacional para tres ciudades colombianas", Desarrollo y Sociedad, N5ㅇ, Bogotá, Universidad de los Andes.

Vignoles, A. y otros (2000), "The relationship between resource allocation and pupil attainment: a review", Documento de Trabajo, № 2, Londres, London School of Economics and Political Science.

Vivas, H., J. B. Correa y J. A. Domínguez (2011), "Potencial de logro educativo, entorno socioeconómico y familiar: una aplicación empírica con variables latentes para Colombia", Sociedad y Economía, № 21, Cali, Universidad del Valle.

Woessmann, L. (2010), "Families, schools and primary-school learning: evidence for Argentina and Colombia in an international perspective", Applied Economics, vol. 42, № 21, Taylor \& Francis.

Woessmann, L. y otros (2007), "School accountability, autonomy, choice, and the level of student achievement. International evidence from PISA 2003", OECD Working Papers, № 13, París, Organización de Cooperación y Desarrollo Económicos (OCDE).

Wooldridge, J. M. (2010), Introducción a la econometría. Un enfoque moderno, Madrid, Cengage Learning. 


\section{Anexo A1}

\section{Estimación del modelo con datos de PISA 2009}

Como análisis de robustez, se replicaron las estimaciones de los cuadros 4 a 6 con datos de PISA 2009. Como se puede observar, los resultados obtenidos no difieren esencialmente de los encontrados para 2012.

\section{Cuadro A1.1}

América Latina (10 países): condicionantes de la adquisición de competencias en lectura, PISA 2009

\begin{tabular}{|c|c|c|c|c|c|c|c|c|c|c|}
\hline & ARG & BRA & $\mathrm{CHL}$ & $\mathrm{COL}$ & CRI & MEX & PAN & PER & URY & VEN \\
\hline \multirow{2}{*}{ constante } & 315,57 & 348,34 & 285,51 & 328,80 & 335,89 & 308,30 & 223,94 & 231,66 & 337,17 & 169,93 \\
\hline & $(23,610)^{\star}$ & $(11,447)^{\star}$ & $(28,735)^{\star}$ & $(17,931)^{\star}$ & $(27,350)^{\star}$ & $(7,893)^{\star}$ & $(88,753)^{\star \star}$ & $(21,815)^{\star}$ & $(11,573)^{\star}$ & $(118,951)$ \\
\hline \multirow{2}{*}{ norepetidor } & 143,21 & 206,51 & 235,64 & 196,66 & 262,58 & 204,91 & 265,79 & 305,64 & 147,03 & 346,66 \\
\hline & $(30,962)^{*}$ & $(16,371)^{*}$ & $(44,379)^{\star}$ & $(24,569)^{\star}$ & $(41,803)^{\star}$ & $(11,486)^{*}$ & $(120,424)^{\star *}$ & $(41,728)^{\star}$ & $(14,192)^{*}$ & $(172,670)^{\star \star}$ \\
\hline \multirow{2}{*}{ sexo } & 22,59 & 4,59 & 0,85 & 3,49 & 6,67 & 8,80 & 3,90 & $-4,87$ & 18,95 & $-16,55$ \\
\hline & $(4,145)^{\star}$ & $(3,266)$ & $(5,030)$ & $(3,866)$ & $(4,687)$ & $(1,930)^{\star}$ & $(7,975)$ & $(5,282)$ & $(2,857)^{\star}$ & $(17,429)$ \\
\hline \multirow{2}{*}{ esfuerzo } & 2,08 & 6,78 & 4,48 & 8,02 & $-3,04$ & 3,62 & $-2,11$ & $-4,04$ & 2,69 & 9,06 \\
\hline & $(3,926)$ & $(2,889)^{\star \star}$ & $(4,039)$ & $(3,659)^{\star \star}$ & $(4,721)$ & $(1,787)^{\star \star}$ & $(7,868)$ & $(4,503)$ & $(2,576)$ & $(9,491)$ \\
\hline \multirow{2}{*}{ disciplina } & 10,61 & 5,74 & 8,30 & 10,58 & $-6,65$ & 4,93 & $-5,23$ & 8,86 & 8,23 & $-6,38$ \\
\hline & $(4,243)^{\star \star}$ & $(3,499)$ & $(4,769)^{\star \star \star}$ & $(5,350)^{\star \star}$ & $(6,194)$ & $(2,395)^{\star \star}$ & $(10,806)$ & $(6,709)$ & $(3,344)^{\star \star}$ & $(11,131)$ \\
\hline \multirow{2}{*}{ libros } & 14,83 & 3,76 & 26,47 & 19,54 & 13,62 & 18,87 & 1,72 & 9,30 & 17,53 & $-1,56$ \\
\hline & $(7,619)^{\star \star \star}$ & * $(7,428)$ & $(6,115)^{\star}$ & $(9,011)^{\star \star}$ & $(11,833)$ & $(4,626)^{*}$ & $(14,197)$ & $(10,965)$ & $(4,432)^{\star}$ & $(15,731)$ \\
\hline \multirow{2}{*}{ educamadre } & 21,48 & 2,22 & 17,02 & 12,93 & $-13,34$ & 14,41 & 3,74 & $-10,33$ & 14,77 & 3,43 \\
\hline & $(5,091)^{\star}$ & $(3,351)$ & $(5,342)^{\star}$ & $(4,303)^{\star}$ & $(6,748)^{\star \star}$ & $(2,052)^{\star}$ & $(10,114)$ & $(9,071)$ & $(3,805)^{\star}$ & $(15,708)$ \\
\hline \multirow{2}{*}{ emppadre } & 9,52 & $-0,89$ & $-9,14$ & 3,63 & $-0,78$ & 7,33 & $-5,39$ & $-5,65$ & $-3,52$ & 21,92 \\
\hline & $(6,767)$ & $(3,374)$ & $(6,431)$ & $(5,068)$ & $(7,134)$ & $(2,728)^{\star}$ & $(14,243)$ & $(5,960)$ & $(4,314)$ & $(23,214)$ \\
\hline \multirow{2}{*}{ publico } & $-28,86$ & $-49,88$ & $-0,56$ & $-21,31$ & $-20,64$ & $-12,15$ & 22,35 & $-6,34$ & $-30,29$ & 44,24 \\
\hline & $(6,415)^{\star}$ & $(6,088)^{\star}$ & $(5,300)$ & $(6,471)^{\star}$ & $(10,110)^{\star \star}$ & $(4,276)^{\star}$ & $(54,604)$ & $(8,586)$ & $(6,365)^{\star}$ & $(61,015)$ \\
\hline \multirow{2}{*}{ STRATIO } & 0,03 & $-0,34$ & $-1,02$ & $-0,95$ & 1,11 & $-0,29$ & 0,60 & $-0,99$ & 0,79 & $-0,54$ \\
\hline & $(0,091)$ & $(0,099)^{\star}$ & $(0,233)^{\star}$ & $(0,1999)^{*}$ & $(0,479)^{\star \star}$ & $(0,029)^{\star}$ & $(0,562)$ & $(0,307)^{\star}$ & $(0,212)^{*}$ & $(0,224)^{\star \star}$ \\
\hline \multirow{2}{*}{ SCMATEDU } & 7,38 & 4,17 & 4,46 & 6,50 & 3,80 & 2,01 & 16,20 & 8,89 & 1,36 & 16,29 \\
\hline & $(2,170)^{*}$ & $(1,663)^{\star \star}$ & $(1,701)^{\star}$ & $(2,040)^{*}$ & $(2,166)^{\star \star \star}$ & $(1,118)^{\star \star \star}$ & $(4,956)^{*}$ & $(2,285)^{*}$ & $(1,269)$ & $(4,329)^{*}$ \\
\hline \multirow{2}{*}{ autonomia } & $-4,96$ & 4,96 & $-3,03$ & 6,39 & $-19,53$ & 5,76 & 5,09 & 13,54 & 0,36 & $-4,89$ \\
\hline & $(4,252)$ & $(2,837)^{\star \star \star}$ & $(3,872)$ & $(3,629)^{\star \star \star}$ & $(5,486)^{\star}$ & $(2,094)^{\star}$ & $(8,141)$ & $(4,604)^{\star}$ & $(2,608)$ & $(12,838)$ \\
\hline \multirow{2}{*}{ SCHSIZE } & 0,00 & 0,00 & 0,01 & 0,00 & 0,00 & 0,00 & 0,00 & 0,01 & 0,00 & 0,02 \\
\hline & $(0,004)$ & $(0,002)$ & $(0,002)^{\star}$ & $(0,001)^{\star}$ & $(0,006)$ & $(0,001)$ & $(0,016)$ & $(0,004)^{*}$ & $(0,003)$ & $(0,010)^{\star \star \star}$ \\
\hline Observaciones & 2485 & 10976 & 3194 & 5866 & 3403 & 27172 & 1950 & 4686 & 3988 & 1660 \\
\hline
\end{tabular}

Fuente: Elaboración propia, sobre la base de datos de la Organización de Cooperación y Desarrollo Económicos (OCDE) sobre la prueba PISA 2009.

Nota: * significativo al 1\%, ${ }^{\star \star}$ significativo al $5 \%,{ }^{\star \star \star}$ significativo al 10\%. Desviaciones estándar robustas entre paréntesis. 


\section{Cuadro A1.2}

América Latina (10 países): condicionantes de la adquisición de competencias en matemáticas, PISA 2009

\begin{tabular}{|c|c|c|c|c|c|c|c|c|c|c|}
\hline & ARG & BRA & $\mathrm{CHL}$ & $\mathrm{COL}$ & CRI & MEX & PAN & PER & URY & VEN \\
\hline \multirow{2}{*}{ constante } & 344,07 & 385,70 & 294,99 & 329,84 & 359,21 & 334,95 & 267,97 & 293,87 & 373,24 & 243,21 \\
\hline & $(16,278)^{\star}$ & $(9,064)^{*}$ & $(26,479)^{\star}$ & $(15,719)^{\star}$ & $(21,377)^{\star}$ & $(7,177)^{\star}$ & $(78,365)^{\star}$ & $(18,268)^{\star}$ & $(9,751)^{\star}$ & $(85,576)^{*}$ \\
\hline \multirow{2}{*}{ norepetidor } & 107,51 & 149,90 & 217,52 & 176,96 & 212,80 & 179,02 & 220,13 & 240,25 & 131,49 & 211,27 \\
\hline & $(23,083)^{\star}$ & $(12,893)^{\star}$ & $(41,318)^{\star}$ & $(21,674)^{\star}$ & $(32,489)^{*}$ & $(10,385)^{\star}$ & $(111,873)^{\star *}$ & $(34,484)^{\star}$ & $(11,980)^{\star}$ & $(124,853)^{\star \star \star}$ \\
\hline \multirow{2}{*}{ sexo } & $-18,72$ & $-33,39$ & $-38,69$ & $-39,02$ & $-31,79$ & $-28,19$ & $-23,10$ & $-39,87$ & $-33,52$ & $-37,60$ \\
\hline & $(3,862)^{*}$ & $(2,579)^{\star}$ & $(4,646)^{*}$ & $(3,349)^{*}$ & $(3,727)^{*}$ & $(1,764)^{*}$ & $(6,926)^{*}$ & $(4,259)^{\star}$ & $(2,478)^{*}$ & $(12,108)^{\star}$ \\
\hline \multirow{2}{*}{ esfuerzo } & $-1,19$ & 3,05 & 1,94 & 8,00 & $-4,74$ & 4,16 & 4,83 & $-2,77$ & $-0,74$ & 2,95 \\
\hline & $(3,427)^{*}$ & $(2,267)$ & $(3,789)$ & $(3,198)^{\star \star}$ & $(3,733)$ & $(1,610)^{\star}$ & $(6,616)$ & $(3,657)$ & $(2,259)$ & $(5,994)$ \\
\hline \multirow{2}{*}{ disciplina } & 8,58 & 4,24 & 8,55 & 10,10 & $-5,29$ & 3,95 & $-1,50$ & 6,62 & 5,66 & 0,01 \\
\hline & $(3,894)^{\star \star}$ & $(2,795)$ & $(4,459)^{\star \star \star}$ & $(4,585)^{\star \star}$ & $(4,974)$ & $(2,159)^{\star \star *}$ & * $(8,707)$ & $(5,343)$ & $(2,873)^{\star \star}$ & $(7,102)$ \\
\hline \multirow{2}{*}{ libros } & 17,87 & 7,72 & 31,09 & 26,57 & 8,22 & 20,94 & 22,08 & 11,12 & 24,99 & 8,50 \\
\hline & $(6,487)^{\star}$ & $(5,869)$ & $(5,883)^{\star}$ & $(7,468)^{\star}$ & $(9,007)$ & $(4,208)^{*}$ & $(14,779)$ & $(8,998)$ & $(4,011)^{\star}$ & $(10,598)$ \\
\hline \multirow{2}{*}{ educamadre } & 15,68 & 2,62 & 15,39 & 13,13 & $-6,21$ & 13,27 & $-6,30$ & $-0,62$ & 14,92 & 20,59 \\
\hline & $(4,314)^{*}$ & $(2,665)$ & $(4,996)^{*}$ & $(3,793)^{\star}$ & $(5,367)$ & $(1,858)^{\star}$ & $(9,299)$ & $(7,291)$ & $(3,368)^{*}$ & $(10,800)^{\star \star \star *}$ \\
\hline \multirow{2}{*}{ emppadre } & 9,93 & 0,21 & $-4,50$ & $-5,64$ & $-9,46$ & 4,19 & $-16,69$ & $-10,26$ & $-2,03$ & 9,75 \\
\hline & $(5,885)^{\star \star \star}$ & $(2,612)$ & $(5,924)$ & $(4,254)$ & $(5,733)^{\star \star \star}$ & $(2,448)^{\star \star \star}$ & * $(13,003)$ & $(4,875)^{\star \star}$ & $(3,709)$ & $(15,496)$ \\
\hline \multirow{2}{*}{ publico } & $-19,86$ & $-65,18$ & 0,40 & $-18,26$ & $-17,75$ & $-10,21$ & 9,92 & $-18,28$ & $-24,62$ & 41,30 \\
\hline & $(5,087)^{*}$ & $(4,861)^{\star}$ & $(4,918)$ & $(5,810)^{*}$ & $(8,043)^{\star \star}$ & $(4,170)^{\star \star}$ & $(49,118)$ & $(7,035)^{*}$ & $(5,493)^{\star}$ & $(42,045)$ \\
\hline \multirow{2}{*}{ STRATIO } & $-0,01$ & $-0,22$ & $-1,54$ & $-0,85$ & 0,89 & $-0,24$ & 0,65 & $-1,33$ & 0,82 & $-0,60$ \\
\hline & $(0,065)$ & $(0,076)^{*}$ & $(0,223)^{\star}$ & $(0,172)^{\star}$ & $(0,377)^{\star \star}$ & $(0,026)^{*}$ & $(0,538)$ & $(0,254)^{\star}$ & $(0,187)^{\star}$ & $(0,142)^{*}$ \\
\hline \multirow{2}{*}{ SCMATEDU } & 10,05 & 2,94 & 4,76 & 6,27 & 5,41 & 3,34 & 14,45 & 7,99 & 3,56 & 14,62 \\
\hline & $(2,013)^{*}$ & $(1,322)^{\star *}$ & $(1,585)^{*}$ & $(1,802)^{*}$ & $(1,751)^{\star}$ & $(1,098)^{*}$ & $(4,004)^{\star}$ & $(1,868)^{*}$ & $(1,103)^{\star}$ & $(2,790)^{*}$ \\
\hline \multirow[b]{2}{*}{ autonomia } & $-4,40$ & 2,37 & 1,88 & 7,09 & $-16,71$ & 5,62 & 3,62 & 8,77 & 3,39 & $-4,34$ \\
\hline & $(3,710)$ & $(2,235)$ & $(3,600)$ & $(3,192)^{\star \star}$ & $(4,283)^{*}$ & $(1,865)^{\star}$ & $(7,572)$ & $(3,751)^{\star \star}$ & $(2,281)$ & $(8,751)$ \\
\hline \multirow{2}{*}{ SCHSIZE } & 0,01 & 0,00 & 0,01 & 0,01 & $-0,01$ & 0,00 & 0,00 & 0,01 & $-0,01$ & 0,03 \\
\hline & $(0,004)^{\star \star \star}$ & $(0,002)$ & $(0,002)^{*}$ & $(0,001)^{*}$ & $(0,004)$ & $(0,000)$ & $(0,016)$ & $(0,003)^{*}$ & $(0,002)^{\star \star}$ & $(0,007)^{\star}$ \\
\hline Observaciones & 1994 & 10976 & 3194 & 5866 & 3403 & 27172 & 1903 & 4686 & 3981 & 1660 \\
\hline
\end{tabular}

Fuente: Elaboración propia, sobre la base de datos de la Organización de Cooperación y Desarrollo Económicos (OCDE) sobre la prueba PISA 2009.

Nota: * significativo al 1\%, ${ }^{\star *}$ significativo al 5\%, ${ }^{\star \star \star}$ significativo al 10\%. Desviaciones estándar robustas entre paréntesis. 
Cuadro A1.3

América Latina (10 países): condicionantes de la adquisición de competencias en ciencias, PISA 2009

\begin{tabular}{|c|c|c|c|c|c|c|c|c|c|c|}
\hline & ARG & BRA & $\mathrm{CHL}$ & $\mathrm{COL}$ & CRI & MEX & PAN & PER & URY & VEN \\
\hline \multirow{2}{*}{ constante } & 317,37 & 390,64 & 318,85 & 367,09 & 374,51 & 338,22 & 149,89 & 274,51 & 347,51 & 242,73 \\
\hline & $(30,387)^{*}$ & $(9,809)^{\star}$ & $(27,390)^{\star}$ & $(15,844)^{\star}$ & $(22,095)^{\star}$ & $(6,718)^{\star}$ & $(169,262)$ & $(18,803)^{\star}$ & $(11,098)^{\star}$ & $(88,688)^{\star}$ \\
\hline \multirow{2}{*}{ norepetidor } & 169,84 & 167,24 & 217,65 & 172,59 & 207,84 & 167,99 & 384,86 & 253,86 & 155,80 & 239,19 \\
\hline & $(48,917)^{\star}$ & $(13,936)^{\star}$ & $(42,761)^{\star}$ & $(21,940)^{\star}$ & $(33,925)^{\star}$ & $(9,701)^{\star}$ & $(237,546)$ & $(35,974)^{*}$ & $(13,325)^{\star}$ & $(129,713)^{\star \star \star}$ \\
\hline \multirow{2}{*}{ sexo } & $-8,61$ & $-22,94$ & $-26,52$ & $-27,50$ & $-23,94$ & $-20,31$ & $-30,00$ & $-26,64$ & $-22,88$ & $-32,67$ \\
\hline & $(5,978)$ & $(2,810)^{*}$ & $(4,768)^{\star}$ & $(3,390)^{\star}$ & $(3,850)^{\star}$ & $(1,708)^{\star}$ & $(13,680)^{\star \star}$ & $(4,485)^{\star}$ & $(2,811)^{*}$ & $(13,220)^{\star \star}$ \\
\hline \multirow{2}{*}{ esfuerzo } & $-2,45$ & 4,53 & 5,12 & 8,00 & $-0,13$ & 4,62 & $-9,72$ & 0,17 & 1,97 & 5,72 \\
\hline & $(4,308)$ & $(2,483)^{\star \star \star}$ & $(3,908)$ & $(3,220)^{\star \star}$ & $(3,885)$ & $(1,575)^{\star}$ & $(11,934)$ & $(3,847)$ & $(2,551)$ & $(6,877)$ \\
\hline \multirow{2}{*}{ disciplina } & $-1,77$ & 4,32 & 8,85 & 6,86 & $-3,25$ & 3,37 & $-6,73$ & 8,21 & 10,76 & 0,21 \\
\hline & $(4,826)$ & $(3,021)$ & $(4,569)^{\star \star \star}$ & $(4,696)$ & $(5,102)$ & $(2,118)$ & $(16,402)$ & $(5,671)$ & $(3,317)^{*}$ & $(7,953)$ \\
\hline \multirow{2}{*}{ libros } & 11,39 & 6,42 & 31,78 & 29,05 & 13,42 & 21,22 & $-1,08$ & 8,36 & 17,62 & 8,91 \\
\hline & $(7,942)$ & $(6,949)$ & $(5,736)^{\star}$ & $(7,525)^{\star}$ & $(9,424)$ & $(4,092)^{\star}$ & $(23,054)$ & $(9,275)$ & $(4,510)^{*}$ & $(11,909)$ \\
\hline \multirow{2}{*}{ educamadre } & 20,35 & 2,93 & 9,76 & 10,94 & $-11,85$ & 16,89 & $-5,46$ & $-11,13$ & 14,74 & 15,69 \\
\hline & $(7,559)^{\star}$ & $(2,918)$ & $(5,091)^{\star \star *}$ & $(3,783)^{\star}$ & $(5,653)^{\star \star}$ & $(1,823)^{\star}$ & $(17,478)$ & $(7,726)$ & $(3,723)^{\star}$ & $(12,015)$ \\
\hline \multirow[b]{2}{*}{ emppadre } & 2,82 & $-0,30$ & $-9,71$ & $-4,81$ & 0,68 & 3,40 & $-15,58$ & $-7,55$ & $-5,15$ & 26,67 \\
\hline & $(8,170)$ & $(2,899)$ & $(6,002)$ & $(4,347)$ & $(5,814)$ & $(2,362)$ & $(24,547)$ & $(5,065)$ & $(4,133)$ & $(16,381)$ \\
\hline \multirow{2}{*}{ publico } & $-17,95$ & $-59,28$ & $-2,15$ & $-26,48$ & $-32,77$ & $-15,08$ & 122,05 & $-14,25$ & $-18,52$ & 15,03 \\
\hline & $(9,101)^{\star \star}$ & $(5,150)^{\star}$ & $(5,015)$ & $(5,556)^{\star}$ & $(8,312)^{\star}$ & $(3,769)^{\star}$ & $(114,428)$ & $(7,347)^{\star \star \star}$ & $(6,090)^{*}$ & $(44,987)$ \\
\hline \multirow{2}{*}{ STRATIO } & 0,23 & $-0,27$ & $-1,25$ & $-1,14$ & 0,74 & $-0,27$ & 1,22 & $-0,81$ & 1,00 & $-0,30$ \\
\hline & $(0,111)^{\star \star}$ & $(0,087)^{*}$ & $(0,226)^{\star}$ & $(0,180)^{\star}$ & $(0,391)^{\star \star \star}$ & $(0,026)^{\star}$ & $(1,198)$ & $(0,261)^{*}$ & $(0,205)^{\star}$ & $(0,163)^{\star \star \star}$ \\
\hline \multirow{2}{*}{ SCMATEDU } & 6,39 & 2,64 & 3,54 & 4,50 & 2,01 & 2,99 & 30,17 & 7,23 & 1,53 & 14,88 \\
\hline & $(2,825)^{\star \star}$ & $(1,392)^{\star \star *}$ & $(1,635)^{\star \star}$ & $(1,765)^{\star \star}$ & $(1,792)$ & $(0,967)^{\star}$ & $(9,193)^{\star}$ & $(1,959)^{*}$ & $(1,263)$ & $(3,193)^{*}$ \\
\hline \multirow{2}{*}{ autonomia } & $-1,06$ & 1,57 & $-0,61$ & 2,57 & $-13,52$ & 7,02 & 8,93 & 10,78 & 0,22 & $-6,03$ \\
\hline & $(5,154)$ & $(2,455)$ & $(3,680)$ & $(3,197)$ & $(4,470)^{*}$ & $(1,851)^{\star}$ & $(12,664)$ & $(3,918)^{\star}$ & $(2,581)$ & $(9,452)$ \\
\hline \multirow{2}{*}{ SCHSIZE } & 0,00 & $-0,01$ & 0,01 & 0,00 & 0,00 & 0,00 & $-0,03$ & 0,01 & $-0,01$ & 0,02 \\
\hline & $(0,006)$ & $(0,002)^{\star \star}$ & $(0,002)^{*}$ & $(0,001)^{\star \star *}$ & $(0,005)$ & $(0,000)$ & $(0,033)$ & $(0,003)^{\star}$ & $(0,003)$ & $(0,008)^{\star \star \star}$ \\
\hline Observaciones & 2011 & 10976 & 3194 & 5866 & 3403 & 27172 & 1699 & 4686 & 3929 & 1660 \\
\hline
\end{tabular}

Fuente: Elaboración propia, sobre la base de datos de la Organización de Cooperación y Desarrollo Económicos (OCDE) sobre la prueba PISA 2009.

Nota: $\quad$ significativo al 1\%, ${ }^{\star \star}$ significativo al $5 \%,{ }^{\star \star \star}$ significativo al 10\%. Desviaciones estándar robustas entre paréntesis. 